\title{
Slug flow models: Feasible domain and sensitivity to input distributions
}

\author{
G.F.N. Gonçalves, R. Baungartner, J.B.R. Loureiro*, A.P. Silva Freire \\ Mechanical Engineering Program (PEM/COPPE/UFRJ), Universidade Federal Do Rio de Janeiro, C.P. 68503, 21941-972, Rio de Janeiro, Brazil
}

\section{A R T I C L E I N F O}

\section{Keywords:}

Horizontal slug flow

Feasible domain

Sensitivity analysis

Unity cell

\begin{abstract}
A B S T R A C T
The present work discusses the feasible and validity domains of unit-cell slug models. The models of Dukler and Hubbard (Ind. Eng. Chem. Fund. 14 (4): 337-347, 1975) and Orell (Chem. Eng. Sci. 60 (5):1371-1381, 2005) are particularly considered as reference models, for they are based on distinct formulation hypotheses. A parameter sensitivity analysis is also carried out to assess the uncertainty in predicted slug length distributions. The work further discusses a kinematic methodology for the estimation of slug length distributions, a problem of great interest in the design and determination of the operational conditions of gas-liquid separators. The results are validated through new experiments and the data of Ujang et al. (Int. J. Multiphase Flow 32 (5): 527-552, 2006). The new experiments introduce data (including the pdf distributions) on pressure gradients, bubble lengths, passage frequency and translational velocity of bubbles. Particle image velocimetry measurements furnish the local mean velocity profiles in the continuous phase of the liquid slug and film.
\end{abstract}

\section{Introduction}

Slug flow is the prevalent flow pattern in many industrial applications. The complexity of slug flows naturally motivated the development of mechanistic models from constraints imposed by physical laws (mass and momentum balances) and some extra empirical correlations (Nicklin et al., 1962; Dumitrescu, 1943; Davies and Taylor, 1950; Wallis, 1969; Dukler and Hubbard, 1975; Nicholson et al., 1978). Normally referred to as unit-cell models, these approximations predict in a simplified but accurate manner the main hydrodynamic parameters of the flow. In literature, the domain of validity of unit-cell models is determined from experimental data or models specially developed to predict flow pattern transition.

As mentioned in Taitel et al. (2000), the above models are very useful for many conditions, but cases are often encountered for which a solution does not exist. The work of Taitel et al. (2000) particularly discusses the case of downward inclined pipes. Four limitations are identified to model application, from both mathematical and physical aspects. In fact, the model deficiencies identified in Taitel et al. (2000) are even found for some gas and liquid flow rates typical of horizontal flow.

The present work studies the mathematical and physical validity domains of unit-cell horizontal slug flow models. The successful application of a particular unit-cell model to flow conditions pertaining to a validity domain naturally demands that all required mathematical operations be justified. For example, fraction denominators and logarithmic terms cannot respectively be zero or have a negative argument.
Physical constraints imposed by the nature of flow parameters must also be satisfied. Lengths and frequency, for instance, cannot be negative numbers.

Here, we discuss the interrelation between domains that are determined from the independent methods - experiments and transition theories - and domains that are determined purely from the requirement that mathematical operations be justified and physical parameters be consistent with their definition. To avoid confusion, the former domains are here called validity domains; the latter domains are called feasible domains. In particular, we show that the validity domains obtained through the independent classical approaches contain large regions where some mathematical operations and physical conditions are not justified. The implication is that models do not furnish solutions in conditions where they are expected to be valid (from the classical point of view) and this is a serious problem in the implementation of engineering prediction software. Clearly, in many instances, models are observed not to cover the entire validity domain predicted by experiments and theories for pattern transition. The true workable domain is therefore the intersection domain of the validity and feasible domains.

Two conceptually different unit-cell models are select for analysis. The model of Dukler and Hubbard (1975) (D\&H) takes into account the shape of the large bubbles and uses a shedding rate relation for prediction of the front propagation velocity, $V_{t}$. The model of Orell (2005) considers cylindrical bubbles and uses an input relation for $V_{t}$.

In the model of Dukler and Hubbard (1975), the passage frequency of unit cells $\left(v_{t}\right)$ is a critical closure parameter. The model of Orell (2005), on the other hand, does not require information on $v_{t}$. However,

\footnotetext{
* Corresponding author.

E-mail address: jbrloureiro@gmail.com (J.B.R. Loureiro).
} 


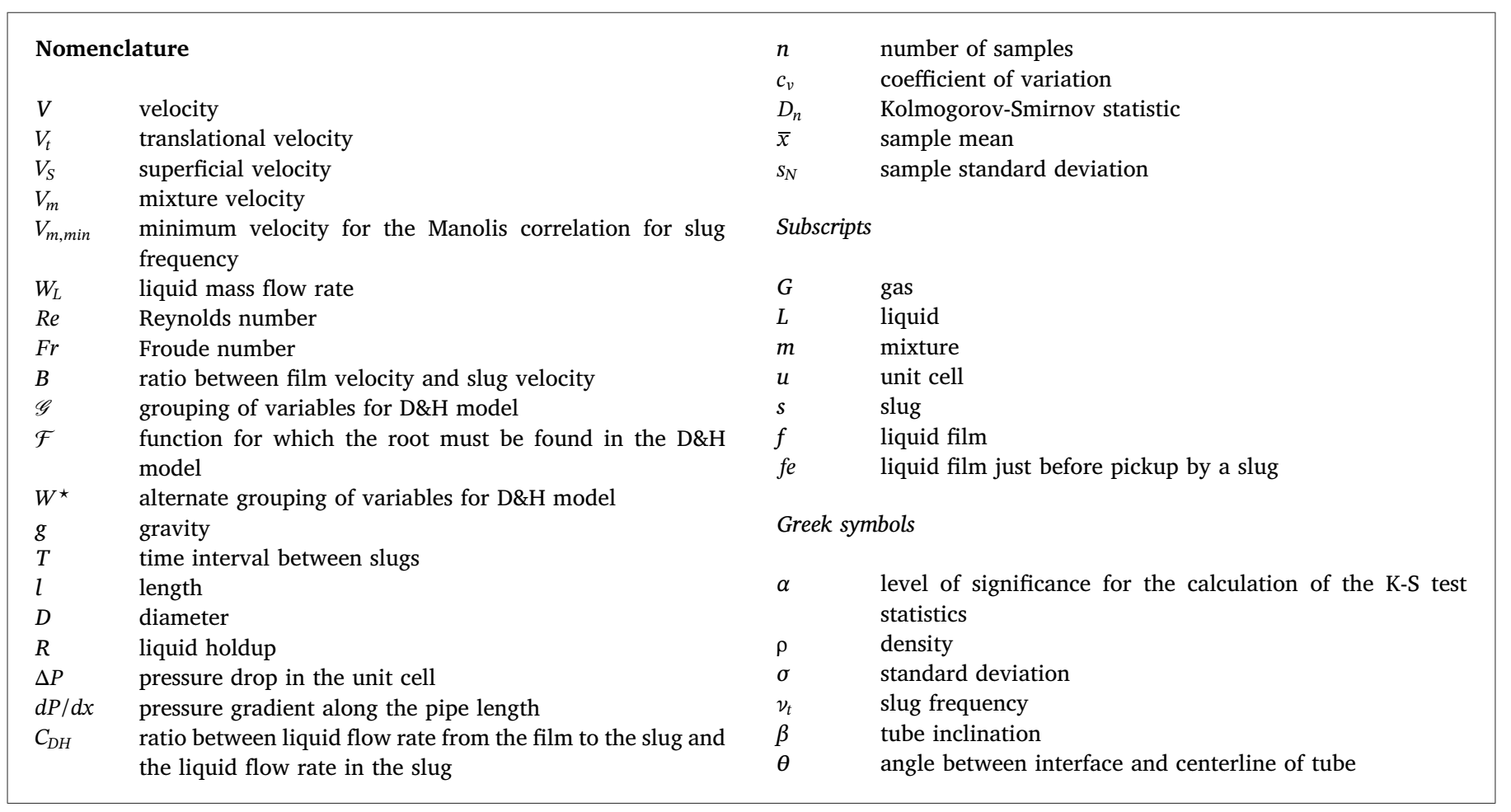

only prediction of the relative lengths of the liquid slug and unit cell, $l_{s} / l_{u}$, is returned. The absolute value of $l_{s}$ (or $l_{u}$ ) is not provided by the model. The implication is that if the model of Orell (2005) is to furnish data on $l_{u}$ an extra closure relation needs to be considered. Of course, since $v_{t}=V_{t} l_{u}^{-1}$, the additional relation might be thought of as a relation for $v_{t}$. In other words, since $V_{t}$ is a fixed modeling parameter of the model of Orell, the specification of $l_{u}$ is equivalent to the specification of $v_{t}$ and vice-versa. The result is that, in fact, $v_{t}$ may be interpreted as a critical closure parameter for both models, as long as $l_{s}$ is an expected output.

Another question of great engineering relevance is: given a known input distribution of $v_{t}$ with a certain coefficient of variation $\left(c_{v}\left(v_{t}\right)\right)$, which values of $c_{v}$ are returned by the unit-cell models to the other flow parameters? Also, how do these values compare with the available experimental data?

In fact, the answer to the above question is the one required by the designers and operators of, e.g., gas-liquid separators. How does one get distributions of the length of the liquid film, $l_{f}$, and how reliable are they? In the second part of the paper, a parameter sensitivity analysis is carried out to answer this question. We show that for the model of Dukler and Hubbard (1975) a value of $c_{v}\left(\nu_{t}\right)=0.6$ corresponds to $c_{v}\left(\Delta P / l_{u}\right)=0.11$ and $c_{v}\left(l_{s} / D\right)=1.2$. To assess how well a given data set follows a specific distribution, the Kolmogorov-Smirnov test statistic is used.

The specific question of obtaining distributions of $v_{t}$ and $l_{s}$ is discussed from the point of view of evolution methods. The essence of these models (see, e.g., Cook and Behnia (2000a)) is a single kinematic wave equation that relates the velocities of the front and the rear of liquid slugs with their own length, and hence determines the translational velocity of a bubble solely as a function of the length of the downstream liquid slug. From a random distribution of slug lengths at the inlet section, the models evaluate the evolution of the probability distribution of $l_{s}$ at any given position. As a corollary, the models yield distributions of $v_{t}$.

Some authors (Ujang et al., 2006; Nydal et al., 1992; Van Hout et al., 2001; Al-Safran et al., 2005) suggest the slug flow parameters $l_{u}$ and $v_{t}$ to be well represented by log-normal distributions. Hence, other relevant questions are: Do evolution models yield log-normal distributions for $l_{u}$ and $v_{t}$ ? Does an input log-normal distribution of $v_{t}$ to a unit-cell model return a log-normal distribution to $l_{u}$ or the other flow parameters?

To help answer all the above questions, a new set of experiments is presented here. The flow conditions are defined through the combination of three liquid and three gas flow rates. Overall, nine flow conditions are discussed. The new data furnishes distributions on $v_{t}, l_{s}, V_{t}$ and $d P / d x$, an information that is important for model validation. Particle image velocimetry measurements furnish the local mean velocity profiles in the continuous phase of the liquid slug and film.

The present manuscript is structured in two parts. First, a study on the feasible and validity domains is conducted (Sections 2 through 5). The relevant aspects of the implementation of the unit cell models of Dukler and Hubbard (1975) and Orell (2005) is presented in Section 2. The evolution model of Cook and Behnia (2000a) is introduced in Section 3. The results for the feasible and validity domains and their comparison with flow pattern maps are shown in Sections 4 and 5, for the models of D\&H and Orell, respectively. In the second part (Sections 6 through 8), new experiments are introduced in Section 6. This database, together with the experimental results of Ujang et al. (2006) are used to study the sensitivity of the D\&H model to input distributions in Section 7. Slug flow predictions with evolution models are discussed in Section 8. The work closes with some critical remarks in Section 9.

\section{Unit-cell models for slug flow prediction}

In the production and transportation of hydrocarbons in horizontal and vertical pipelines, slug flow is certainly the dominant flow configuration. Be it due to natural occurrence or to artificially introduced enhancing production techniques, flow patterns resulting from a sequence of long bubbles followed by liquid slugs permeated by small bubbles are largely encountered in the oil industry.

Unfortunately, the modeling of slug flow presents some serious difficulties due to its chaotic features. Any casual observation of slug flows shows that the characteristic lengths of the long bubbles and liquid slugs appear to be randomly distributed in time and space.

A classical manner of treating slug flow is to postulate the existence of a typical cell that repeats itself moving down a pipe. Provided there 
exists a reference frame where the liquid and gas phases are considered to travel with about the same velocity and to attain a fully developed state, disregard of the flow randomness is justifiable to a certain point (Fabre and Liné, 1992). A steady state hypothesis results in many simplifications that can then be used to develop a less complicate - but reliable - working model. The simplifying assumptions can give origin to models where the cells have a single fixed length or models where a stochastic distribution of the cell length is considered.

A theory of vertical slug flow was advanced by Nicklin et al. (1962) after some early observations by Dumitrescu (1943) and Davies and Taylor (1950). The theory basically discussed the motion of large bubbles moving in vertical liquid streams.

Wallis (1969) was possibly the first to discuss horizontal and vertical slug flows from the point of view of a unit cell. In his analysis, the bubble and liquid phase dynamics were modelled so that expressions for the void fraction and pressure drop became available. This work was followed by a comprehensive model proposed by Dukler and Hubbard (1975). Compared to the previous formulations, the model of D\&H was much more detailed. The authors discussed the conditions of slug flow, its initiation and dissipation.

Nicholson et al. (1978) suggested improvements to the model of Dukler and Hubbard (1975); in particular, new equations were proposed for the average translational velocity of the unit cell and the liquid fraction in the slug, an input quantity.

More recently, unit cell models have been given more simplified formulations. The work of Orell (2005), e.g., incorporates a modified pressure loss term as suggested by Cook and Behnia (2000b). The effect of gas entrainment in the slug on the wall shear stress is accounted for with the introduction of an effective viscosity. This effect is carried over to the definition of the Reynolds number employed in the evaluation of the friction coefficient.

The models of Dukler and Hubbard (1975) and Orell (2005) were chosen for discussion here due to their inherent differences in formulation. In addition, the model of Dukler and Hubbard (1975) is considerably more sophisticated from the mathematical point of view so that the analysis on feasible domain, sensitivity and uncertainty is naturally enriched.

In flow configurations with changes in slope (hilly terrain), the distribution of slug length and the maximum slug length must be predicted through transient models. To that end, models that track the front and back of individual slugs can be used to evaluate their characteristic lengths. For details on this approach, refer to the models of AlSafran et al. (2004) and Zhang et al. (2003), which resort to the first principles for flow description. The unsteady velocity behavior of newly initiated slugs was investigated in Al-Safran et al. (2013).

\subsection{Comments on flow pattern map and slug frequency models}

The topology of the geometry of two-phase flows is normally expressed in terms of flow pattern maps. However, because there exists in the literature some disagreement in regard to the terms that best describe any observed phase distribution, different flow maps can be found by different authors. The flow pattern maps of Mandhane et al. (1974) and Taitel and Dukler (1976) are typical examples (Fig. 1).

In the following we refer to the map of Mandhane et al. (1974) due to its large acceptance in the literature.

Based on empirical and mechanistic arguments, different methods to predict the slug frequency $v_{t}$ have been proposed by several authors. An obvious problem with most formulations is the difficulty to incorporate all variables that mostly affect slug frequency, including slug initiation mechanisms, the superficial velocities of the liquid and gas phases, the pipe diameter and inclination, and the properties of the fluids. Zabaras (2000) and Al-Safran, (2009) reviewed the correlations of several authors and in the process proposed their own.

Here, three correlations are briefly introduced.

Heywood and Richardson (1979). The correlation of Heywood and
Richardson (1979) was developed for air-water slug flows in a $42 \mathrm{~mm}$ diameter pipe. The correlation is expressed in dimensional parameters (SI units) and is given by

$v_{t}=0.0364 \frac{V_{L S}}{V_{m}}\left(\frac{2.02}{D}+\frac{V_{m}^{2}}{g D}\right)^{1.06}$

where $V_{L S}$ is the liquid superficial velocity, $V_{m}$ is the mixture velocity, $g$ is the acceleration of gravity and $D$ is the pipe diameter.

Manolis et al. (1995). The correlation of Manolis et al. (1995) introduces a correction on the correlation of Gregory and Scott (1969) to better represent low frequency slug flow. The equation in SI units can be cast as

$v_{t}=0.0037 \frac{V_{L}}{g D}\left(\frac{V_{m, \min }^{2}+V_{m}^{2}}{V_{m}}\right)^{1.8}$

where $V_{m, \min }=5 \mathrm{~ms}^{-1}$.

Zabaras (2000). The formulation of Gregory and Scott (1969) also lays ground for the correlation of Zabaras (2000). Zabaras analized 339 data points to propose a correlation that incorporates the effects of pipe slope. The correlation of Zabaras is expressed in English units and is given by

$v_{t}=0.0226\left(\frac{V_{L S}}{g D}\right)^{1.2}\left[\frac{19.75}{V_{m}}+V_{m}\right]^{1.2}\left[0.836+2.75 \sin ^{0.25}(\beta)\right]$

where $\beta$ is the inclination angle measured from the horizontal.

\subsection{The model of Dukler and Hubbard (1975)}

The idealized slug model of Dukler and Hubbard (1975) consists of a long bubble traveling over a liquid film that is followed by a liquid plug with a strong mixing region at the front; the latter structure is referred to in literature as the liquid slug (or simply "slug"). Since in the film region pressure is essentially constant, pressure losses are confined to the slug region where they can be considered to arise from two effects: the acceleration of the slow moving liquid film to slug velocity and the pressure loss required to overcome the wall shear stress in the main part of the slug (Dukler and Hubbard, 1975).

The general configuration of the model of Dukler and Hubbard (1975) is presented in Fig. 2, together with some of the relevant

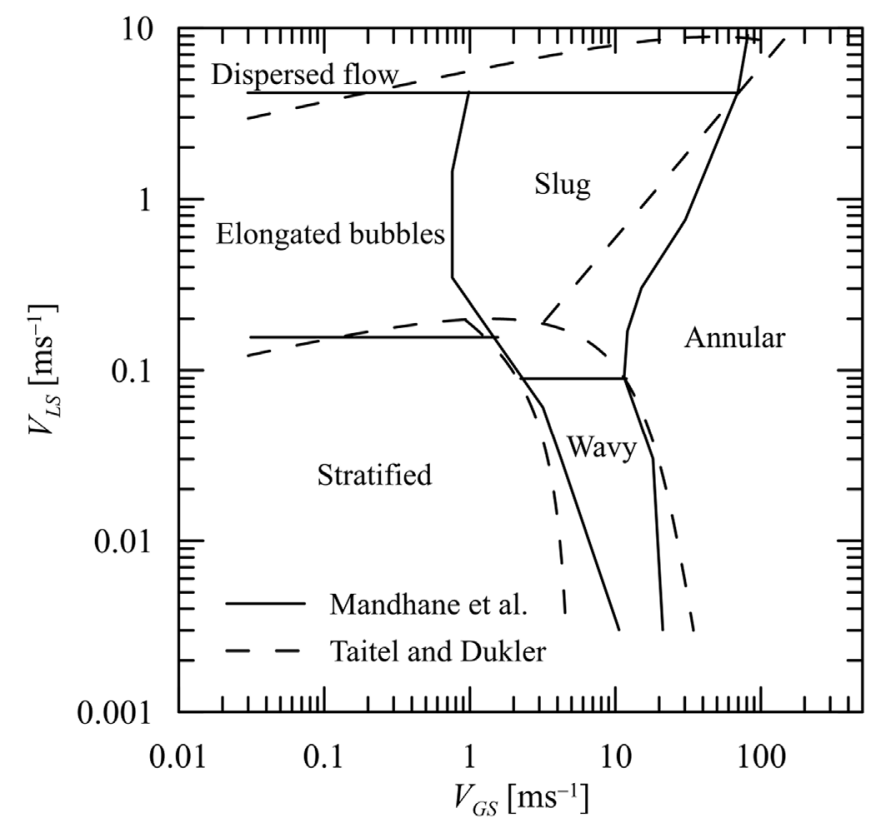

Fig. 1. The two-phase horizontal flow pattern maps of Mandhane et al. (1974) and Taitel and Dukler (1976) for pipes with $D=0.05 \mathrm{~m}$. 


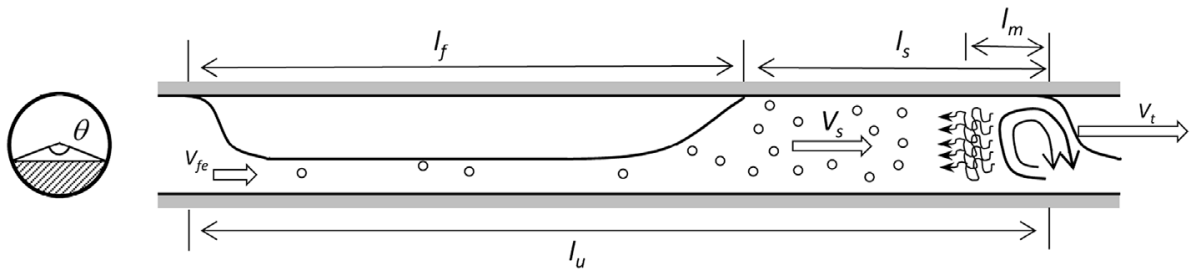

Fig. 2. Unit cell model structure according to Dukler and Hubbard (1975). parameters. For a complete description of the model, the reader is referred to the original source or alternatively to Bandeira et al. (2017). In particular, the present notation is based on Bandeira et al. (2017). Note that difficulties (to be discussed in the present contribution) found in the implementation of the model of Dukler and Hubbard (1975) have led authors to introduce the original formulation in different forms. Here, we follow the original developments, as described in Bandeira et al. (2017).

The system of equations introduced by Dukler and Hubbard (1975) must be solved to determine $l_{f}$ and $R_{f e}$, the liquid holdup of the film just before pick-up. These equations, however, can be rearranged to result in a single equation to $\theta_{f e}(\theta$ is the angle that subtends the liquid film; see Dukler and Hubbard (1975)).

The final form of the equation to $\theta_{f e}$ is

$\mathcal{F}\left(\theta_{f e}\right)=\int_{\theta_{f e}}^{\theta_{S}} W^{\star}(\theta) d \theta-\mathscr{G}\left(\theta_{f e}\right)=0$,

with

$$
W^{\star}(\theta)=\frac{\frac{C_{D H}^{2} R_{S}^{2}}{\left(\frac{\theta-\sin \theta}{2 \pi}\right)^{2}}+\frac{1}{F r}\left(\frac{\frac{\pi}{2}\left(\frac{\theta-\sin \theta}{2 \pi}\right) \sin \frac{\theta}{2}+\sin ^{2} \frac{\theta}{2} \cos \frac{\theta}{2}}{1-\cos \theta}-\frac{1}{2} \cos \frac{\theta}{2}\right)}{0.0791\left(\frac{B(\theta-\sin \theta)}{\theta} R e_{s}\right)^{-0.25} B^{2} \frac{\theta}{\pi}+\frac{\left(\frac{\theta-\sin \theta}{2 \pi}\right)}{F r} \sin \beta},
$$

and

$$
\begin{aligned}
\mathscr{G}\left(\theta_{f e}\right)= & \frac{V_{s}}{v_{t} D\left[R_{s}-\left(\frac{\theta_{f e}-\sin \theta_{f e}}{2 \pi}\right)\right]}\left\{\frac{W_{L}}{\rho_{L} A V_{s}}-\left(\frac{\theta_{f e}-\sin \theta_{f e}}{2 \pi}\right)\right. \\
& \left.+C_{D H}\left[R_{s}-\left(\frac{\theta_{f e}-\sin \theta_{f e}}{2 \pi}\right)\right]\right\}-\frac{V_{t}}{v_{t} D} .
\end{aligned}
$$

The feasible domain of the model of Dukler and Hubbard (1975) is studied next in connection with parameter $\mathcal{F}\left(\theta_{f e}\right)$.

\subsection{The model of Orell (2005)}

The model of Orell (2005) is a simplification of the model introduced by Taitel and Barnea (1990), which considers uniform film thickness. The model also takes into account the increase of the apparent viscosity in the liquid slug due to the presence of air bubbles. The general configuration of the model together with some of the relevant parameters are shown in Fig. 3.

The details of the model can be obtained in Orell (2005) or else in Bandeira et al. (2017). The model is based on a non-linear system of equations that has to be solved for the variables: $\theta, V_{f}, V_{G}, l_{s} / l_{u}$. In fact, the system of equations can be written in terms of $\theta$, so that just one transcendental equation needs to be solved numerically.

The model evaluates the pressure gradient directly, without the need to specify the pressure drop per unit cell. As such, no information about frequency or slug length is necessary.

\section{Evolution model, the model of Cook and Behnia (2000a)}

The model of Cook and Behnia (2000a) is based on the work of Barnea and Taitel (1993). Both models determine the statistical distribution of slug lengths from an empirical expression that defines the rate of collapse of short slugs as a function of their lengths. The models of Barnea and Taitel (1993) and Cook and Behnia (2000a) differ through the specification of distinct evolution laws. Cook and Behnia (2000a) used their own experimental data whereas Barnea and Taitel (1993) fitted the data of Moissis and Griffith (1962). The model is purely evolutionary. Once the translational velocities of the front and rear of randomly distributed slugs are assigned through the working empirical expressions, the motion of the slug can be described through a simple marching process. The models at no instant resort to the first principles; thus, no dynamic equation of motion is solved.

The details of the model can be obtained directly from Cook and Behnia (2000a). Basically, the evolution of a slug is described by the displacement of the slug front $(F)$ and rear $(R)$ through propagation equations. A slug is said to collapse as $R$ approaches $F$ and the number of each slug behind it decreases by one (Cook and Behnia, 2000a). The results of Barnea and Taitel (1993) and of Cook and Behnia (2000a) show that for large enough distances from the entrance section, the distribution of the lengths of the slugs is insensitive to the initial random distribution.

\section{Feasible domain of the Dukler and Hubbard (1975) model}

The model of Dukler and Hubbard (1975) is examined so as to have its feasible domain determined.

Of course, flow pattern maps only express the expected configuration to be found in flows with specified gas and liquid superficial velocities. They do not provide information on the passage interval of long bubbles, which is an input parameter of great importance to mechanistic models.

Thus, as presented in literature, flow pattern maps are invariant with respect to slug frequency. An implicit assumption in their construction is that given the gas and liquid superficial velocities and the pipe diameter and inclination, a certain slug frequency is fixed. However, given the different formulations found in literature for $v_{t}$, the input data corresponding to a well defined point (location) in a given flow pattern map may be associated to different values of $v_{t}$, which may

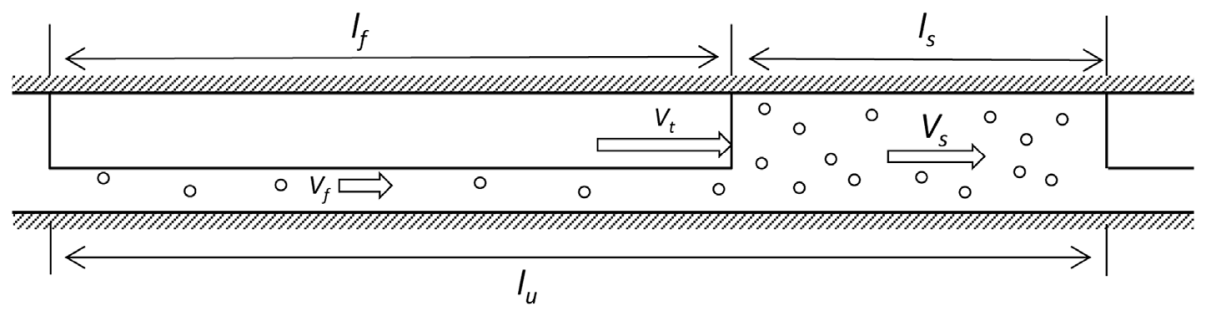

Fig. 3. Unit cell model structure according to Orell (2005). 
or may not result in justified mathematical operations. Indeed, the feasible domain of the system of equations that compose the models requires that all mathematical operations be justifiable and these operations are sensitive to the specification of $v_{t}$.

For the D\&H model three non-conformities are observed:

1 Failure in finding a real root to Eq. (4), a mathematical constraint. 2 Zero or negative value to the liquid slug length: $l_{s} \leq 0$, a physical constraint.

3 Length of the mixing vortex region higher than the total length of the liquid slug: $l_{s}<l_{m}$, a physical constraint.

The sensitivity of the feasible domain of the D\&H model to changes in $v_{t}$ is illustrated in Fig. 4 for two slug frequencies, $v_{t}=0.1$ and 10 $\mathrm{s}^{-1}$. This is a purely mathematical exercise, conceived to show how the model responds to input datum on $v_{t}$. In the region of elongated bubbles the model accepts inputs that may vary an order of magnitude and still furnishes feasible solutions. In the slug flow region the model may fail against over predicted estimations of $v_{t}$, but returns output for under predicted guesses. What Fig. 4 illustrates is the sensitivity of the feasible domain to choices of $v_{t}$ obtained from the several available correlations. Expressions that for a given flow condition yield a lower $v_{t}$ result in a larger feasible domain.

The feasible domain of the D\&H model with the input of $v_{t}$ given by Eq. (3) is shown in Fig. 5. This figure also shows the distribution of the non-conformities $1,2,3$. The feasible domain includes large portions of the regions of stratified and dispersed flows. However, a large portion of experimentally observed slug flow region is out of the range of the model due to non-conformities 2 and 3.

The behavior of the function $\mathcal{F}\left(\theta_{f e}\right)$, Eq. (4), for four flow conditions is shown in Fig. 6. These conditions are representative of the region of valid formulation (slug flow (a)), and the three typical regions where roots cannot be found (the regions of stratified flow (b), dispersed flow (c) and wavy flow (d)). In the dispersed flow region, $\mathcal{F}\left(\theta_{f e}\right)$ is always positive, so that no root can be determined. In the regions of stratified and wavy flows $\mathcal{F}\left(\theta_{f e}\right)$ is always negative.

The sensitivity of the feasible domain to other slug flow frequency correlations is shown in Fig. 7, where results are illustrated for the correlations of Heywood and Richardson (1979) and Manolis et al. (1995). All three feasible domains bear much likeness, but differences

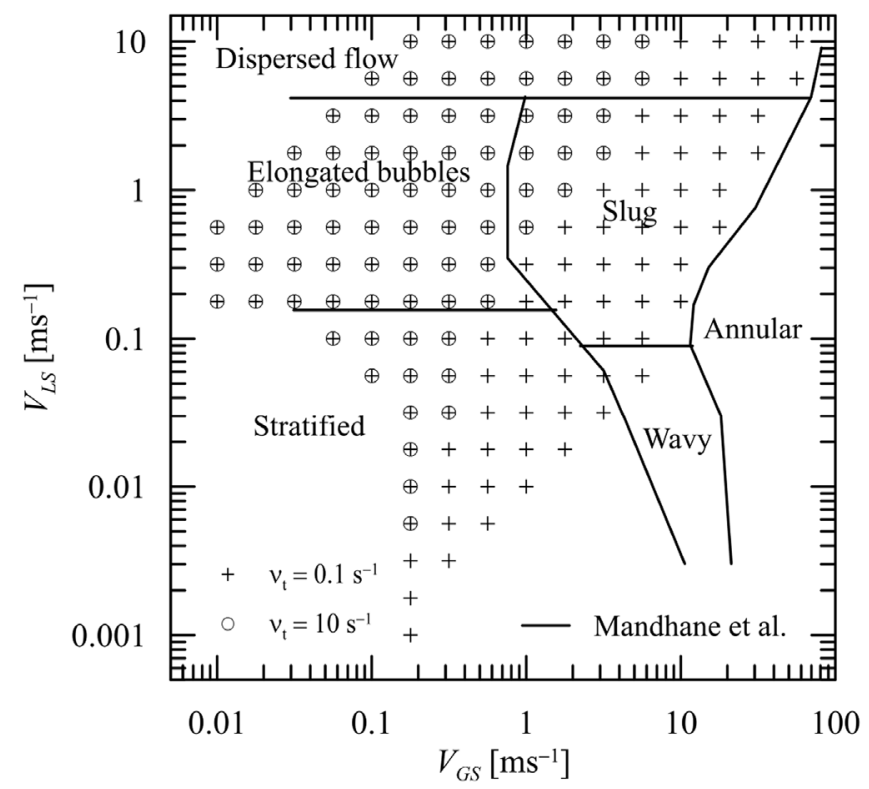

Fig. 4. Comparison between the feasible domain of the model of Dukler and Hubbard (1975) and the flow pattern map of Mandhane et al. (1974) for two slug frequencies, $v_{t}=0.1$ and $10 \mathrm{~s}^{-1}$.

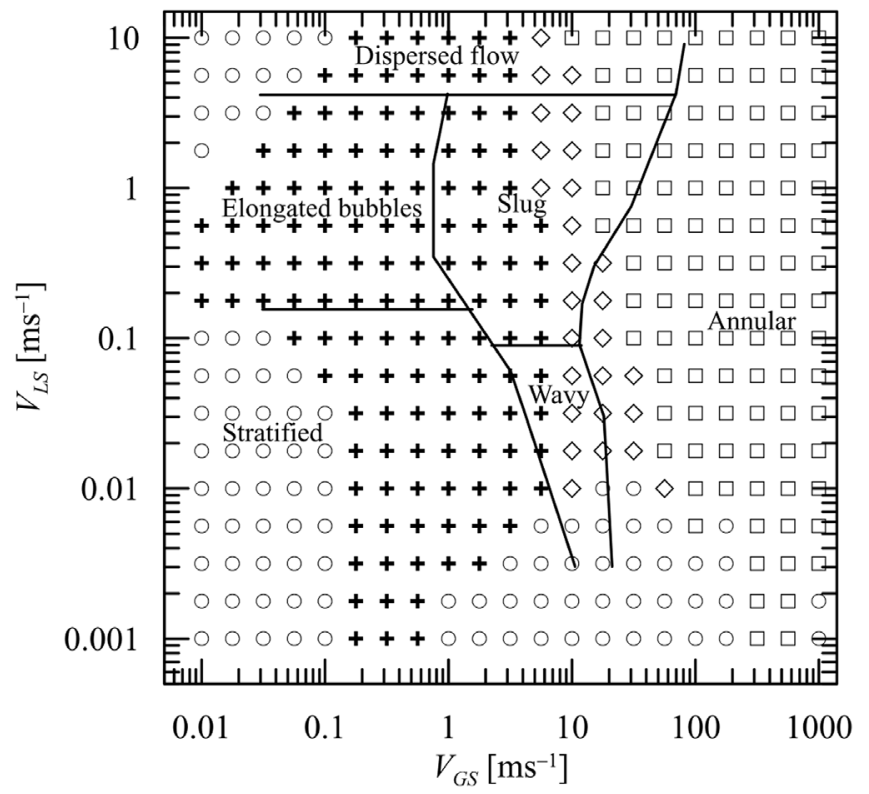

Fig. 5. Assessment of the feasible domain of the model of Dukler and Hubbard (1975) according to the slug frequency expression of Zabaras (2000). Crosses: valid region; 1 circles: no root; 2 boxes: $l_{s} \leq 0$; 3 diamonds: $l_{s}<l_{m}$.

are noticeable. The domain obtained through the correlation of Manolis et al. (1995) is the largest. None of the correlations cover the entire slug flow domain, they all fail for $V_{G S}>6 \mathrm{~ms}^{-1}$.

\section{Feasible domain of the Orell model}

Even the simplified model of Orell (2005) suffers from some nonconformities. In fact, the two identified non-conformities are related to the length of the liquid slug and can be summarized as:

1 Liquid slug length greater than the unit cell length or negative: $l_{s} / l_{u}$ $<0$ or $l_{s} / l_{u}>1$, two physical constraints.

The feasible domain of the Orell model is shown in Fig. 8. The top left area of the region of elongated bubbles - much in the same way as the D\&H - is not covered by the model. However, and unlike the D\&H model, the following features are observed:

- In a large portion of the region of annular flow feasible results are obtained.

- A less pronounced (as compared to the model of D\&H) but still large region of feasible solution is noted in the region of stratified flow.

Figs. 5 and 8 illustrate how different the feasible domain of models can be depending on their constitutive hypotheses.

\section{Experiments}

\subsection{Experimental facilities and instrumentation}

To discuss the sensitivity of the Dukler and Hubbard (1975) model to input distributions and the appropriateness of the Cook and Behnia (2000a) model to generate distributions of $v_{t}$, a new set of experiments is presented here. As previously mentioned, distributions of $v_{t}, l_{f}$ and results for $\Delta P$ are provided.

A general overview of the horizontal multiphase flow loop is presented in Fig. 9. The test facility consists of an acrylic pipe with total length of $12 \mathrm{~m}$ and internal diameter of $44.2 \mathrm{~mm}$. A volumetric pump was used to transport water at a constant flow rate. An air compressor 

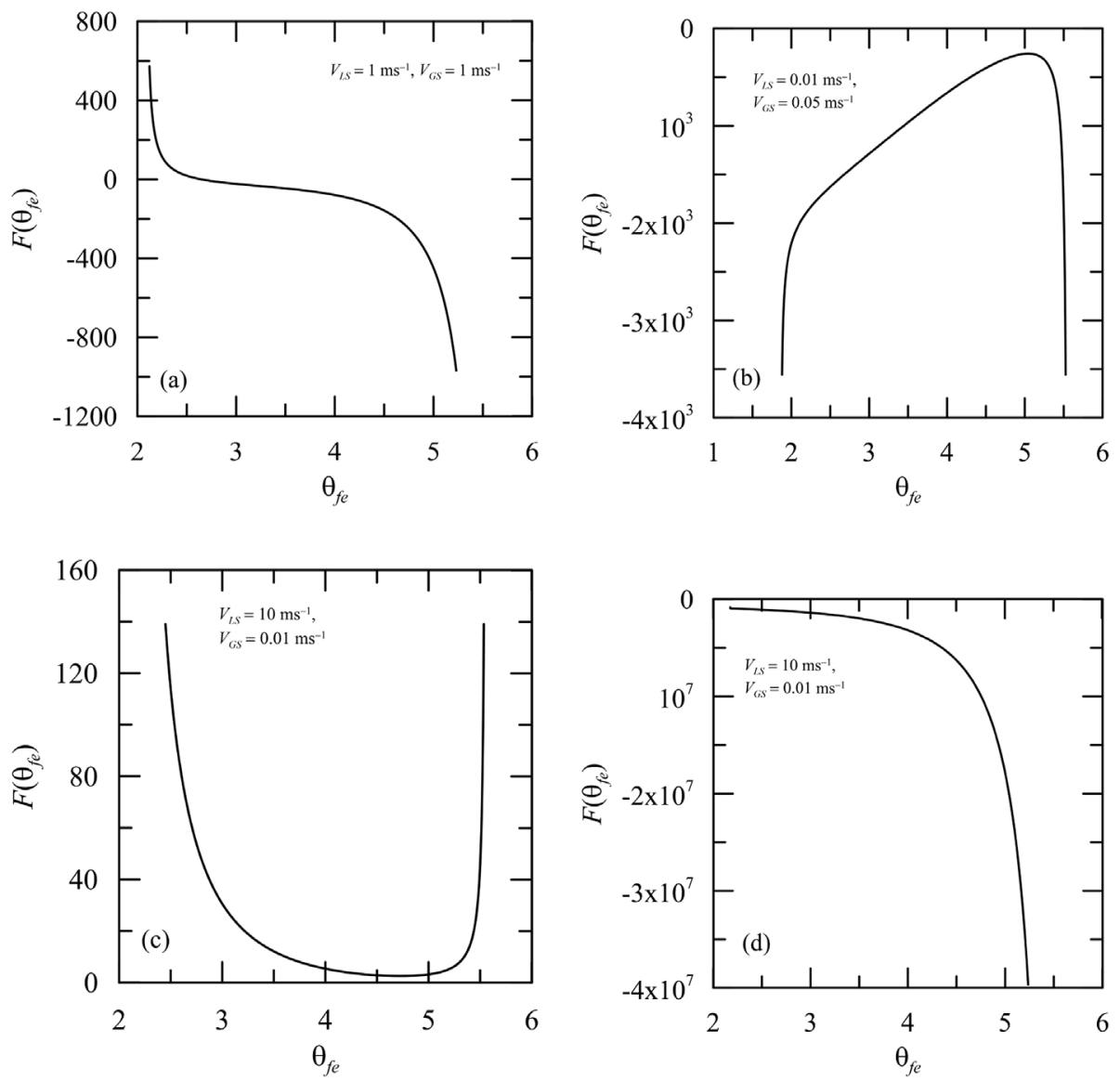

Fig. 6. Behavior of the function $\mathcal{F}\left(\theta_{f e}\right)$, Eq. (4), for the flow conditions: (a) slug flow, (b) stratified flow, (c) dispersed flow, (d) wavy flow.

(Schulz SRS 60) injected dry air into the system through a T-junction at the pipe inlet. The liquid flow rate was controlled through an electromagnetic flow meter (Endress Hauser Promag 10P40), which has an overall uncertainty of $0.5 \%$ of the reading. The gas flow rate was measured with a vortex flow meter (Techmeter FLP04-G2NA) which has an overall uncertainty of $3 \%$ of the reading. Absolute pressure (Rosemount $800 \mathrm{PSI}$ ) and temperature (Siberius KT300) were measured to determine the air properties at the flow condition. A differential pressure transducer (Endress Hauser Deltabar PMD55), with an overall uncertainty of $0.2 \%$ of the upper range limit, was used to measure the pressure gradient along the pipe through a total of twenty pressure taps. All experiments were performed at an ambient temperature of about $22.0^{\circ} \mathrm{C}$. The superficial liquid and gas velocities varied in the respective intervals $[0.72,1.81] \mathrm{ms}^{-1}$ and $[0.2,0.8] \mathrm{ms}^{-1}$.

The observation sections were enclosed by rectangular acrylic boxes; the space between the flat walls and the pipe was filled with deionized water to minimize optical distortions.

The tested gas and liquid flow rates are shown in Table 1.

The characteristics of the dispersed and continuous phases of the flow were determined through the Dantec Dynamics Shadow Sizer System (SSS) and Particle Image Velocimetry (PIV). The present procedures are improved versions of the methodologies described in Magalhaes et al. (2013) and Matamoros et al. (2014).

Bubble characteristics, including length, translational velocity and
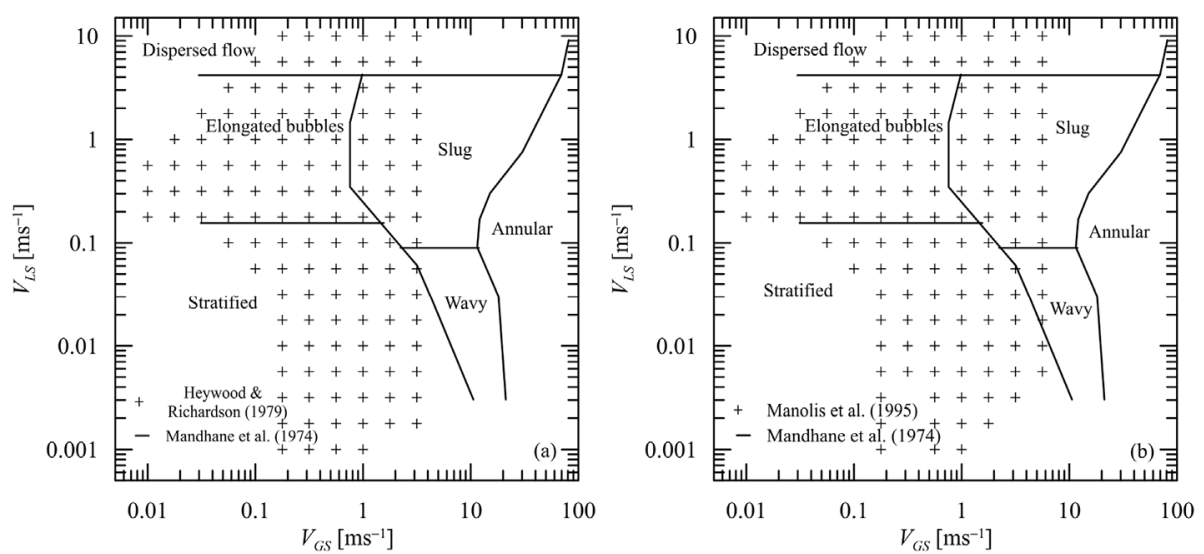

Fig. 7. Sensitivity of the feasible domain of the model of Dukler and Hubbard (1975) to different slug frequency correlations. (a) Heywood and Richardson (1979); (b) Manolis et al. (1995). 


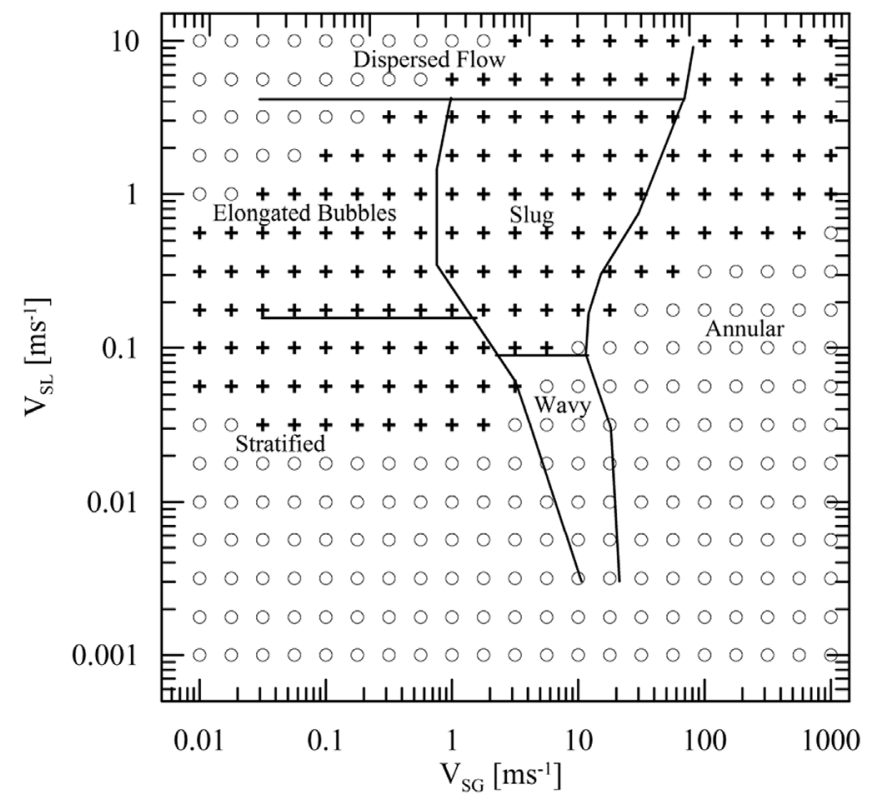

Fig. 8. Assessment of the feasible domain of the model of Orell (2005). Crosses: valid region; circles: $l_{s} / l_{u}<0$ or $l_{s} / l_{u}>1$.

frequency, were measured with the Shadow Sizer System. Images were simultaneously acquired at Stations 1 and 2 (Fig. 9) through two SpeedSense M310 cameras $(1280 \times 1024$ pixels, $3260 \mathrm{fps})$ fitted with lenses AF D Micro-Nikkor $60 \mathrm{~mm} f / 2.8$. Two constellation LED systems were used with light diffusers to provide adequate background illumination. The image illumination and lens focus were adjusted to provide a high contrast between the bubble contour and the background. The size of the field of view was measured and related to the camera pixels by a calibration procedure.

For system synchronization and image acquisition, the software Dynamic Studio of Dantec Dynamics Inc. was used (version 2015a). Image processing was made with a dedicated algorithm written in the software Wolfram Mathematica $^{\mathrm{TM}}$. A series of image processing operations, including subtraction of the mean background and binarization were applied to make the contour of the bubbles sharply defined. Bubble reconstruction was made with the tracking method described in Matamoros et al. (2014). Two sets of 5000 images acquired at a rate of $100 \mathrm{~Hz}$ were obtained for the measurement of bubble statistics. The contour detection algorithm was used to identify the successive positions of the bubble nose in a sequence of frames. The bubble velocity was calculated by measuring the nose displacement along the field of view at the corresponding elapsed time. Velocity was estimated from a least-square fit. Considering each measured bubble velocity constant, the evaluation of the time interval between the bubble nose and tail furnishes the bubble length. The passage frequency of long bubbles was given by the inverse of the elapsed time between subsequent detected
Table 1

Gas and liquid flow rates used in the experiments.

\begin{tabular}{lll}
\hline Experiment & $V_{L S}\left[\mathrm{~ms}^{-1}\right]$ & $V_{G S}\left[\mathrm{~ms}^{-1}\right]$ \\
\hline T1 & 0.72 & 0.27 \\
T2 & 0.72 & 0.49 \\
T3 & 0.72 & 0.78 \\
T4 & 1.27 & 0.22 \\
T5 & 1.27 & 0.42 \\
T6 & 1.27 & 0.68 \\
T7 & 1.81 & 0.21 \\
T8 & 1.81 & 0.43 \\
T9 & 1.81 & 0.65 \\
\hline
\end{tabular}

noses. A detailed description of the methodology used in the present work can be found in Matamoros et al. (2014).

Measurement uncertainties have been evaluated according to the procedure recommended by the Guide to the Expression of Uncertainty in Measurement (JCGM, 2010). The combined standard uncertainty, obtained from the square root of the sum of variances estimated from sources of type A (aleatory) and type B (systematic), is multiplied by the coverage factor to calculate the expanded or overall uncertainty. Since the effective degrees of freedom is higher than 150, the coverage factor chosen for the expression of an interval around the mean value that expresses a 95\% level of confidence was 1.96 . The overall relative uncertainty for the passage frequency, bubble velocity and bubble length are $1.1 \%, 1.3 \%$ and $6.2 \%$, respectively.

Simultaneous 2D PIV and Shadow Sizer Technique have been used at Station 2, following the procedure introduced by Nogueira et al. (2003). The continuous phase is seeded with fluorescent Rhodamine particles, which scatter light a wavelength of around $590 \mathrm{~nm}$. The LED background illumination was covered with an orange diffuser in order to shed light at approximately the same wavelength of the fluorescent particles. A red filter is fitted in the camera lens to allow the passage of wavelengths above $570 \mathrm{~nm}$, blocking the green laser reflections emitted at $532 \mathrm{~nm}$.

The synchronization between the SpeedSense M310 camera, the NdYAG double-pulsed laser and the LED is done so that each frame is simultaneously exposed to both light sources. The acquired images thus contain the seed particles and a well-defined bubble shape information.

\subsection{Slug flow development length}

The general flow pattern observed in the experiments is shown in Fig. 10. The mixing caused by injection of air through the tee junction results in flow patterns where many small bubbles remain dispersed in the upper part of the liquid slug for high mixture velocities.

To investigate the degree of development of the flow for the several gas and water flow rates, measurements were conducted at stations located 4500 and $9000 \mathrm{~mm}$ away from the entrance $(L / D=100$ and 200 respectively). For a given train of bubbles, their number, velocities and lengths were assessed. The synchronized counting system considers

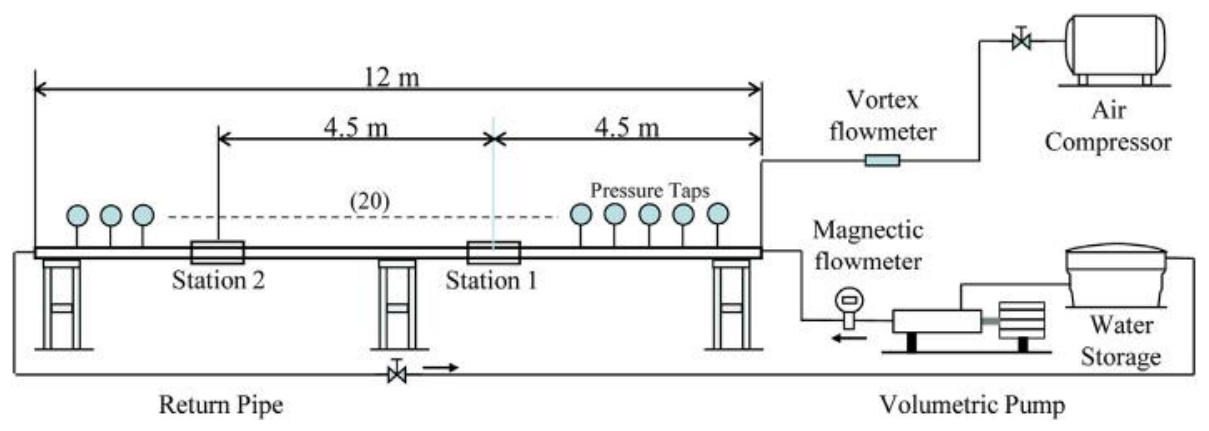

Fig. 9. Schematic diagram of the multiphase flow loop. 


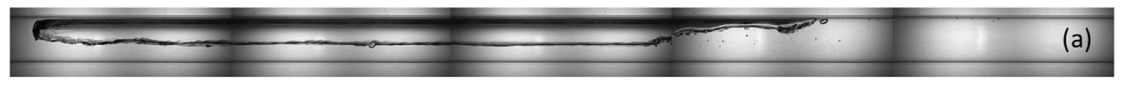

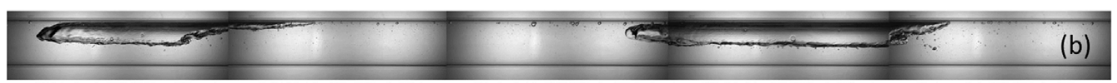

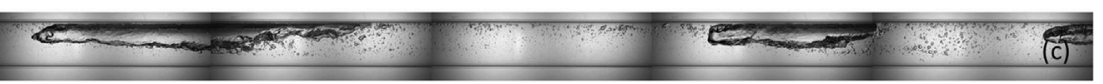

Fig. 10. Air-water slug flow patterns for: (a) $V_{L S}=0.72 \mathrm{~m} / \mathrm{s}$ and $V_{G S}=0.27 \mathrm{~m} / \mathrm{s}$, (b) $V_{L S}=1.27 \mathrm{~m} / \mathrm{s}$ and $V_{G S}=0.22 \mathrm{~m} / \mathrm{s}$, (c) $V_{L S}=1.81 \mathrm{~m} / \mathrm{s}$ and $V_{G S}=0.43 \mathrm{~m} / \mathrm{s}$.

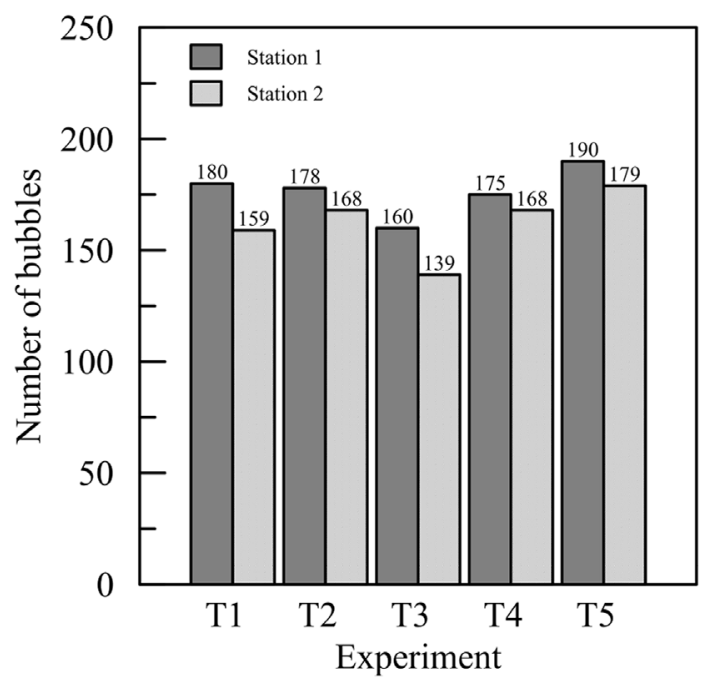

Fig. 11. Number of recorded bubbles at positions 4500 and $9000 \mathrm{~mm}$ downstream of the inlet, for five experimental conditions.

the estimated translational velocity of the bubbles and a correlation algorithm. Fig. 11 shows the average number of registered bubbles at both positions for five experimental conditions. Changes in the number of bubbles were of the order of $7.1 \%( \pm 3.6 \%)$. The flow was thus relatively stable at position $9000 \mathrm{~mm}$.

The above results agree with the study of Ujang et al. (2006). The pipe length and diameter for the experiments of Ujang et al. (2006) were respectively 37,000 and $78 \mathrm{~mm}$ and the inlet conditions were so controlled as to initiate slugs from stratified flows. In this case, the accepted formation mechanism is wave growth. Once formed, the growth, stabilization or reduction in size of a slug depends on the balance between the amount of liquid picked up from the preceding film and the amount of liquid shed at its rear. The superficial liquid and gas velocities in the experiments of Ujang et al. (2006) ranged respectively in the intervals $[0.22,0.61] \mathrm{ms}^{-1}$ and $[2.27,2.55] \mathrm{ms}^{-1}$. For most conditions, a stable slug frequency was observed downstream of the position $L / D=90$. In some extreme conditions, stabilization was

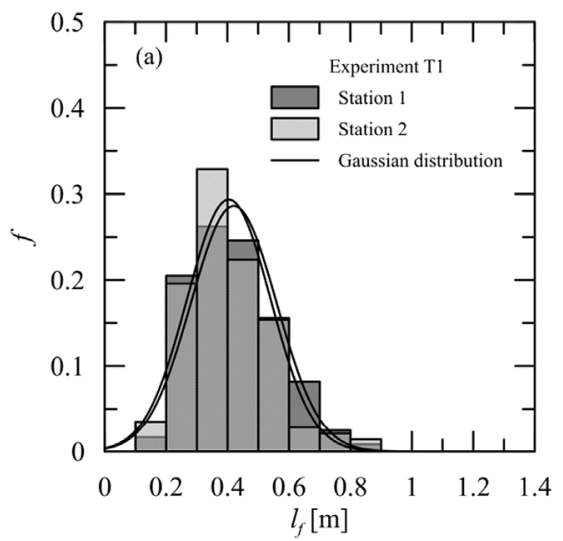

always obtained for $L / D>150$. Note that, for the present experiments, the high mixing caused by the air injection into the main pipe through a $\mathrm{T}$-junction shortens the required length for slug formation.

The distributions of the lengths and velocities of bubbles at the two measuring positions are shown in Figs. 12 and 13. The reduction in the number of bubbles between measuring positions is a result of bubble coalescence, provoked by the existence of liquid slugs that are shorter than the minimum stable length. The small number of coalescing bubbles means that the distributions of long bubble lengths vary very little from one position to the other. Figs. 12 and 13 show that the length and velocity distributions are almost identical for all conditions. The conclusion is that the flow is very near to an equilibrium (stationary) condition.

\subsection{Global flow parameters: $d P / d x, l_{f}, v_{t}$ and $V_{t}$}

Fig. 14 showns the pressure gradient, film length, passage frequency of bubbles and translational velocity for all tested conditions. The following trends (some of them evident) are readily observed:

1. The increase of $V_{L S}$ or $V_{G S}$ increases $d P / d x$ as expected.

2. The increase of $V_{L S}$ decreases $l_{f}$. The increase in $V_{G S}$ increases $l_{f}$.

3. The increase of $V_{L S}$ increases $\nu_{t}$. The increase in $V_{G S}$ has little effect on $v_{t}$.

4. $V_{t}$ increases with increasing $V_{L S}$ or $V_{G S}$.

Fig. 14 is important, since it shows the quantitative behavior of the most important flow parameters. The maximum bubble and liquid slug lengths observed were respectively 1.45 and $0.94 \mathrm{~m}$ (for different flow conditions, $\mathrm{T} 1$ to $\mathrm{T} 9$ ).

For the sake for completeness, the local mean velocities of the continuous phase in the slug and film regions are shown in Fig. 15.

\section{Sensitivity of the Dukler and Hubbard (1975) model}

The response of the model of Dukler and Hubbard (1975) to input frequency distributions is discussed next. In the present section, the analysis is focused on the influence that the input frequency distribution exerts on the predicted distributions furnished by the model, e.g., the length of the liquid slug $\left(l_{s}\right)$ and the pressure gradient.

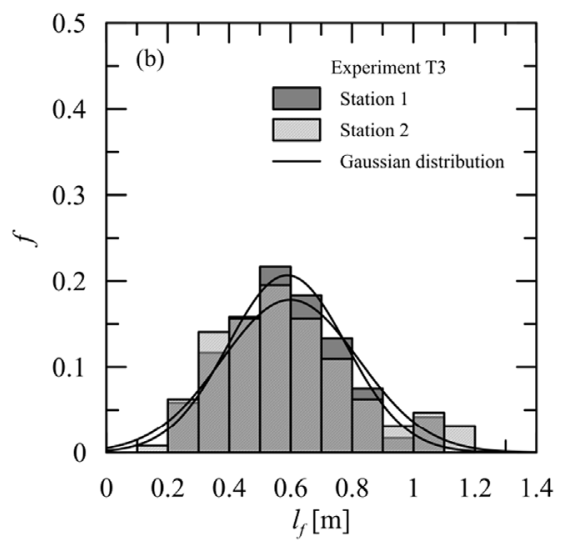

Fig. 12. The distribution of the lengths of bubbles at positions 4500 and $9000 \mathrm{~mm}$ for conditions $\mathrm{T} 1$ and $\mathrm{T} 3$. 

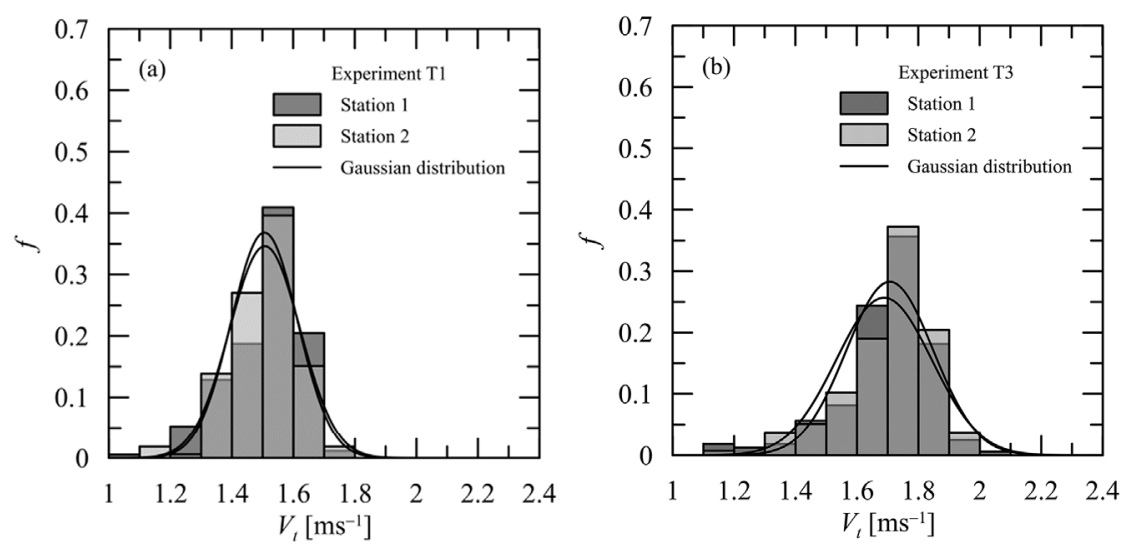

Fig. 13. The distribution of the velocities of bubbles at positions 4500 and $9000 \mathrm{~mm}$ for conditions T1 and T3.

In addition, this section also investigates the influence of the input coefficient of variance on the dispersion of the resulting calculated variables. With this respect, the mean, the variance and the coefficient of variance of a given data set were evaluated, respectively, through the relations:

$\mu=\bar{x}=\frac{1}{N} \sum_{i=1}^{N} x_{i}$

$\sigma=s_{N}=\sqrt{\frac{1}{N} \sum_{i=1}^{N}\left(x_{i}-\bar{x}\right)^{2}}$,

and

$c_{v}=\frac{s_{N}}{\bar{x}}$.
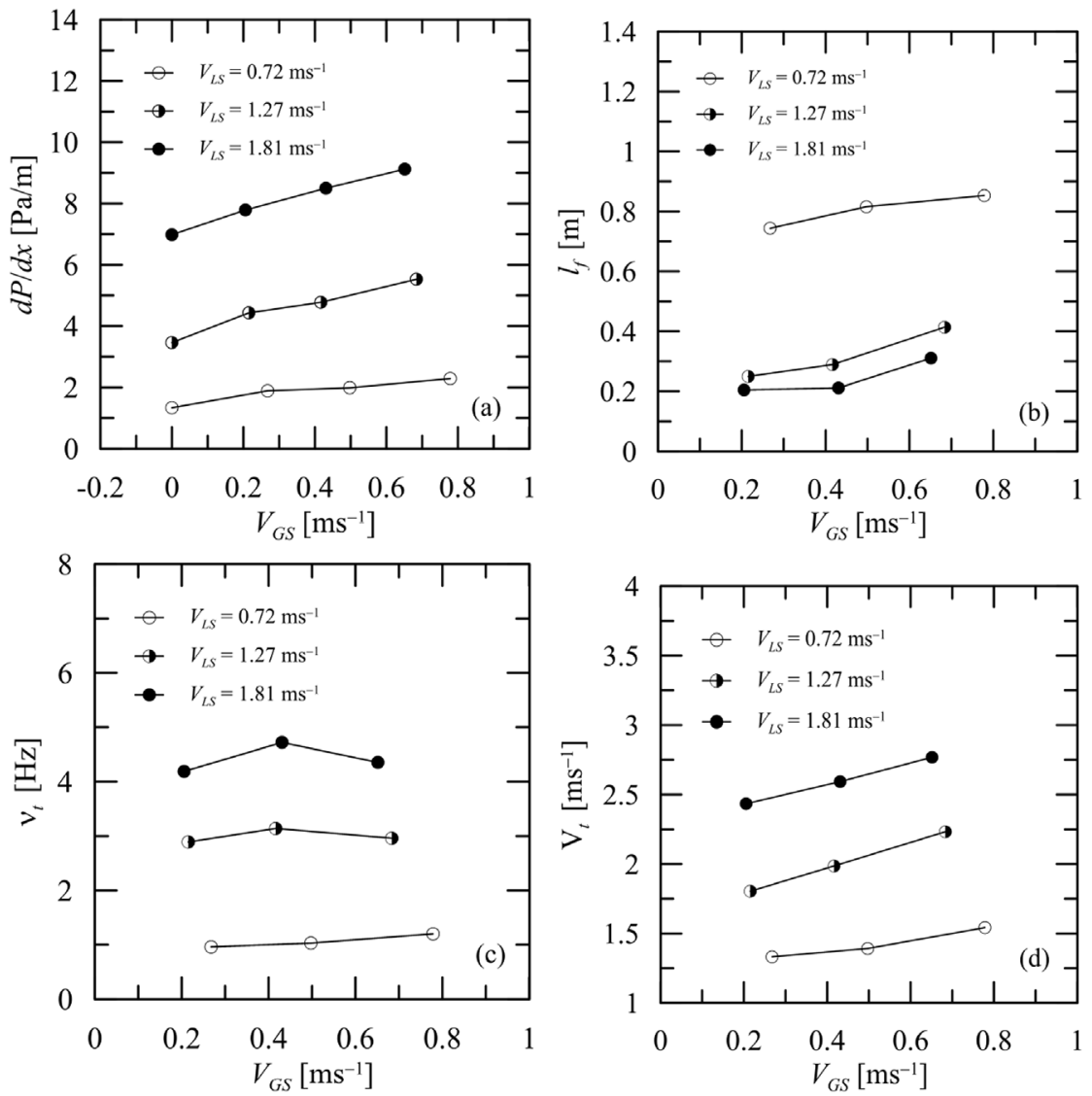

Fig. 14. Total pressure gradient $(d P / d x)$, film length $\left(l_{f}\right)$, passage frequency of bubbles $\left(\nu_{t}\right)$ and translational velocity $\left(V_{t}\right)$ for all tested conditions. 

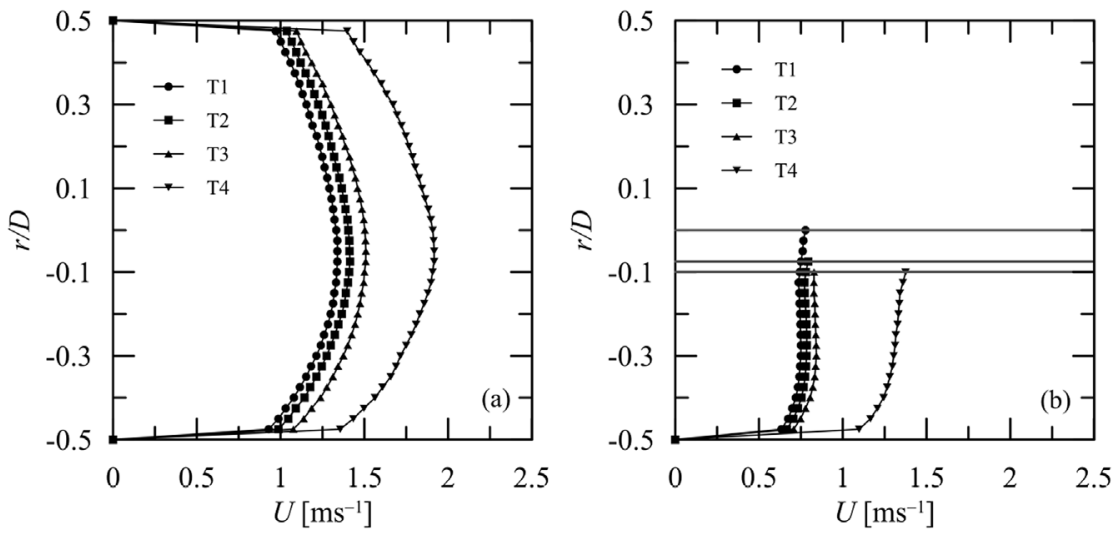

Fig. 15. Local mean velocity profiles in the continuous phase of the slug and film. Experimental conditions according to Table 1.

Table 2

PDF distributions used in the description of liquid slug lengths.

\begin{tabular}{|c|c|c|c|}
\hline & PDF & Parameters & Reference \\
\hline Log-normal & $\frac{1}{x \sqrt{2 \pi} \sigma} \exp ^{-\frac{(\ln x-\mu)^{2}}{2 \sigma^{2}}}$ & $\mu, \sigma$ & $\begin{array}{l}\text { Van Hout et al. (2001), } \\
\text { Brill et al. (1981), } \\
\text { Nydal et al. (1992) }\end{array}$ \\
\hline Inverse Gaussian & $\left(\frac{\lambda}{2 \pi x^{3}}\right)^{1 / 2} \exp \frac{-\lambda(x-\mu)^{2}}{2 \mu^{2} x}$ & $\mu, \lambda$ & $\begin{array}{l}\text { Dhulesia et al. (1991), } \\
\text { Al-Safran et al. (2005) }\end{array}$ \\
\hline Gamma & $\frac{\beta^{-\alpha} x^{\alpha-1} \exp ^{-x / \beta}}{\Gamma(\alpha)}$ & $\alpha, \beta$ & Nieckele et al. (2013) \\
\hline
\end{tabular}

lengths are shown in Table 2.

To decide how well a given data set follows a specific distribution, the Kolmogorov-Smirnov test was used. The Kolmogorov-Smirnov test statistic is defined by
$D_{n}=\max \left|F(x)-F_{o}(x)\right|$.

If $F(x)$ is the theoretical population cumulative distribution and $F_{0}(x)$ is the observed cumulative step-function of a sample, then the distribution of this supremum is known and is independent of $F(x)$, if $F(x)$ is continuous (Massey, 1951).

The critical value, $D_{\alpha}(n)$, depends on the sample size $(n)$ and on the level of significance $(\alpha)$ such that the probability $P[\max$ $\left.\left|F_{0}(x)-F(x)\right|>D_{\alpha}(n)\right]=\alpha$. At the $95 \%$ confidence level $(\alpha=0.05)$ and large sample sizes $(n>35)$, the critical value is $1.36 / \sqrt{n}$. If the test statistic $\left(D_{n}\right)$ is smaller than the critical value for a given level of significance, the hypothesis that the true distribution is $F(x)$ is not rejected. $D_{\alpha}(n)$ can be considered as a margin surrounding the theoretical distribution. If the distance between the observed and the theoretical distribution is larger than this margin at any point $\left(D_{n}>D_{\alpha}(n)\right)$, the null hypothesis that samples come from $F(x)$ is rejected.
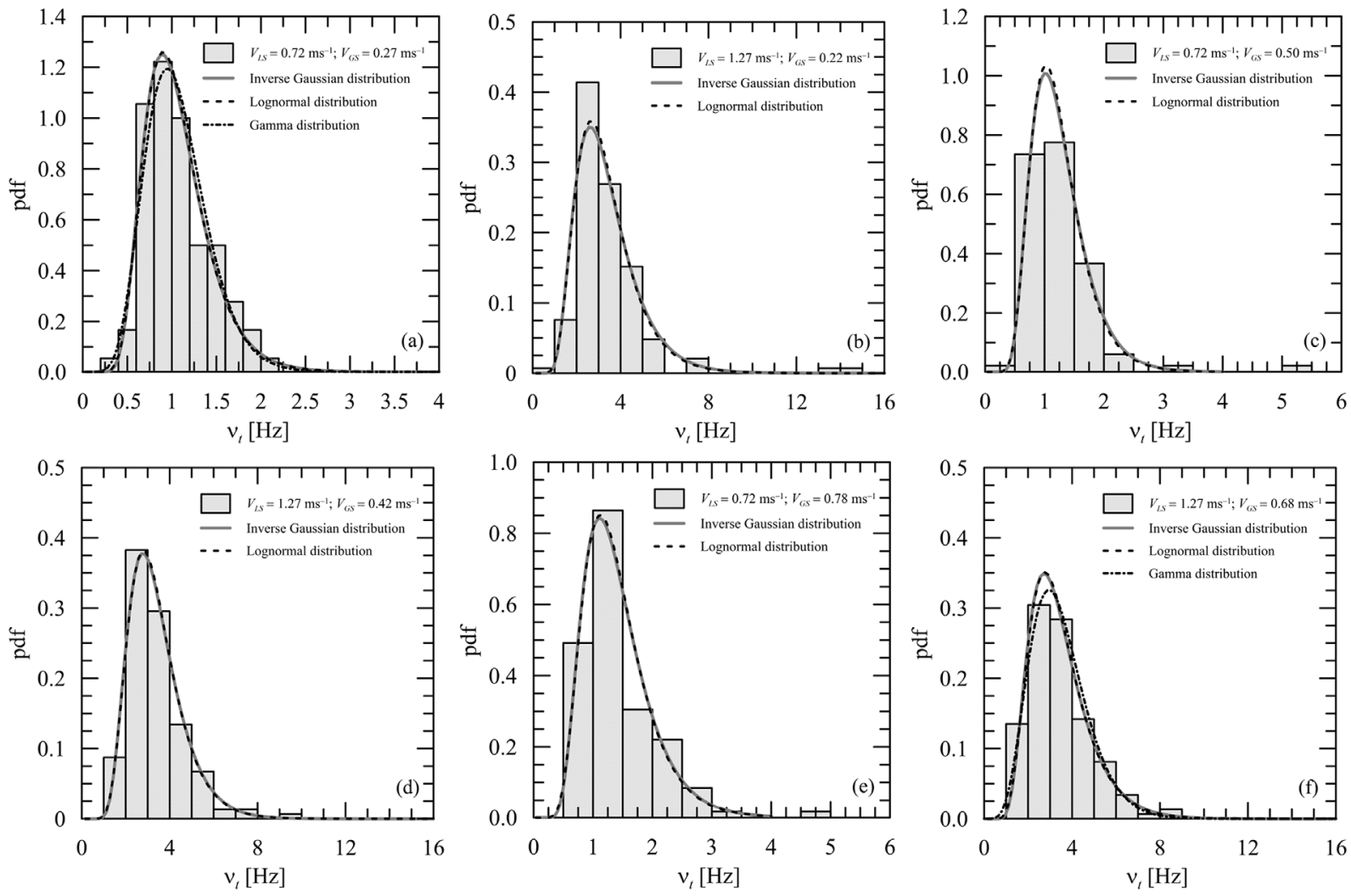

Fig. 16. Distributions of $v_{t}$ (present experiments). 
Table 3

Kolmogorov-Smirnov test statistics of the fitted distributions to $v_{t}[\mathrm{~Hz}]$ (present experiments).

\begin{tabular}{|c|c|c|c|c|c|c|c|c|}
\hline \multicolumn{2}{|l|}{ Exp. Cond. } & \multicolumn{3}{|l|}{$v_{t}$} & \multicolumn{4}{|c|}{ Kolmogorov-Smirnov Test } \\
\hline$V_{L S}\left(\mathrm{~ms}^{-1}\right)$ & $V_{G S}\left(\mathrm{~ms}^{-1}\right)$ & $\mu$ & $\sigma$ & $c_{v}$ & Log-normal & Normal & Gamma & Inverse Gaussian \\
\hline 0.72 & 0.27 & 1.06 & 0.36 & 0.34 & 0.06 & 0.11 & 0.08 & 0.06 \\
\hline 0.72 & 0.50 & 1.25 & 0.58 & 0.47 & 0.07 & 0.15 & 0.10 & 0.08 \\
\hline 0.72 & 0.78 & 1.41 & 0.61 & 0.43 & 0.07 & 0.15 & 0.10 & 0.08 \\
\hline 1.27 & 0.22 & 3.36 & 1.69 & 0.50 & 0.07 & 0.16 & 0.10 & 0.07 \\
\hline 1.27 & 0.42 & 3.38 & 1.29 & 0.38 & 0.06 & 0.12 & 0.08 & 0.06 \\
\hline 1.27 & 0.68 & 3.44 & 1.37 & 0.40 & 0.05 & 0.08 & 0.06 & 0.05 \\
\hline 1.81 & 0.21 & 4.86 & 1.97 & 0.41 & 0.06 & 0.13 & 0.08 & 0.06 \\
\hline 1.81 & 0.43 & 5.37 & 1.90 & 0.35 & 0.05 & 0.10 & 0.07 & 0.05 \\
\hline 1.81 & 0.65 & 5.03 & 2.73 & 0.54 & 0.07 & 0.18 & 0.10 & 0.08 \\
\hline
\end{tabular}
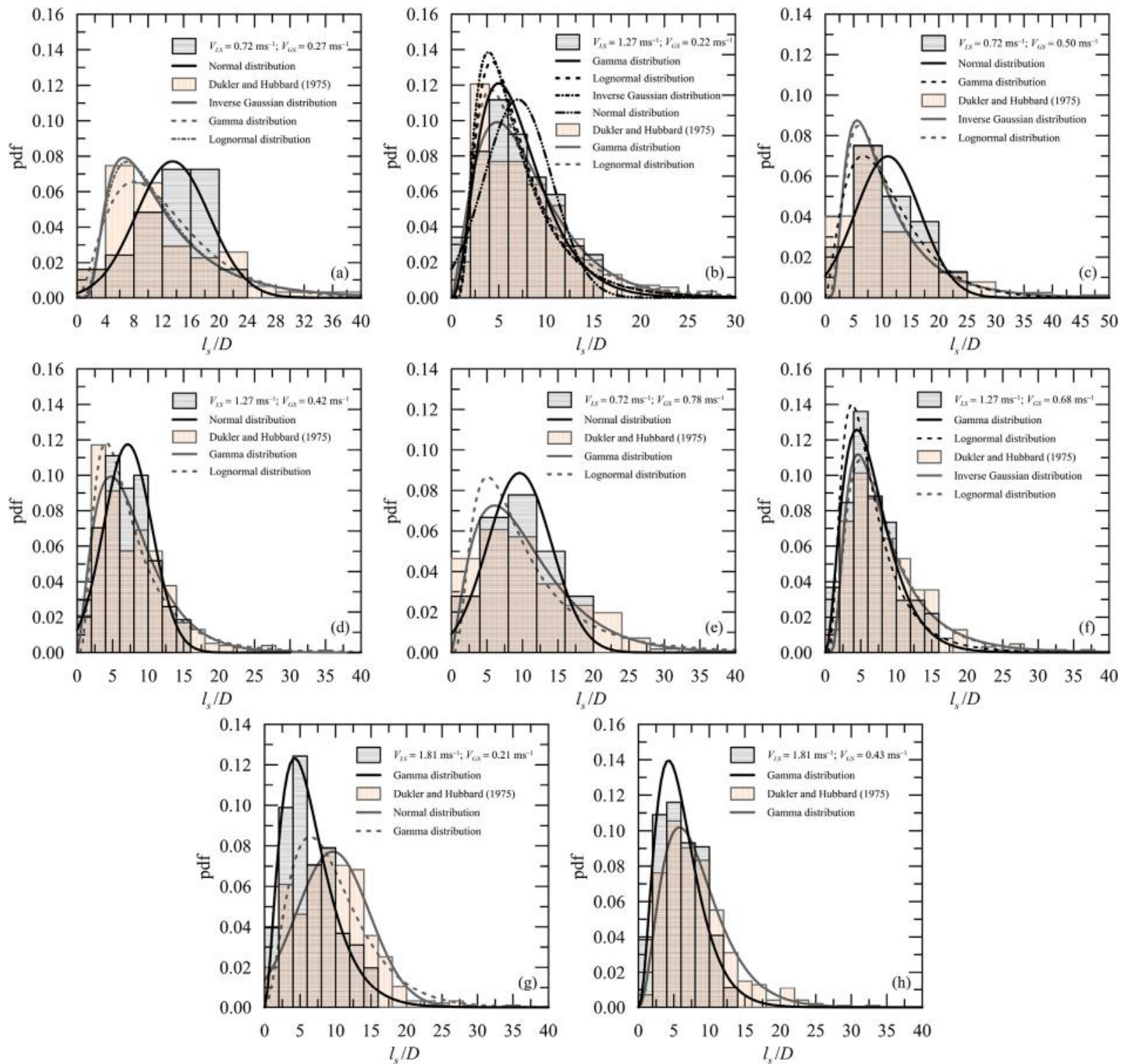

Fig. 17. Best-fitted distributions of $l_{s} / D$ : experiments and predictions (D\&H model) at $x=9 \mathrm{~m}$ from the inlet position.

7.1. Sensitivity of the Dukler and Hubbard (1975) model to input distributions of $\nu_{t}$

The present experimental data cover eight different conditions with superficial velocities ranging from $V_{L S}=0.72 \mathrm{~ms}^{-1}$ to $1.81 \mathrm{~ms}^{-1}$ and $V_{G S}$ $=0.21 \mathrm{~ms}^{-1}$ to $0.78 \mathrm{~ms}^{-1}$. The measured input frequencies $(x=9 \mathrm{~m})$ are shown in the histograms of Fig. 16. In fitted distributions, the continuous line indicates the best fitted distribution, whereas the dashed and dot-dashed lines denote the second and third distribution that were also not rejected by the Kolmogorov-Smirnov test.

An assessment of the goodness-of-fit for four types of distribution is shown in Table 3 . The distributions that were not rejected at the $5 \%$ 
Table 4

Comparison between the experimental values of the mean, standard deviation and coefficient of variance of slug length data $\left(l_{s} / D\right)$ and predicted values (D\&H model).

\begin{tabular}{llllllll}
\hline Exp. Cond. & & Exp. $l_{S} / D$ & \multicolumn{3}{c}{ Predicted $l_{S} / D$ from D\&H } \\
\hline$V_{L S}\left(\mathrm{~ms}^{-1}\right)$ & $V_{G S}\left(\mathrm{~ms}^{-1}\right)$ & $\mu_{\exp }$ & $\sigma_{\exp }$ & $c_{v_{\exp }}$ & $\mu_{D H}$ & $\sigma_{D H}$ & $c_{v_{D H}}$ \\
\hline 0.72 & 0.27 & 13.45 & 5.26 & 0.39 & 12.25 & 7.57 & 0.62 \\
0.72 & 0.50 & 11.02 & 5.81 & 0.53 & 11.05 & 7.65 & 0.69 \\
0.72 & 0.78 & 9.60 & 4.55 & 0.47 & 10.48 & 6.71 & 0.64 \\
1.27 & 0.22 & 7.02 & 3.58 & 0.51 & 7.85 & 5.01 & 0.64 \\
1.27 & 0.42 & 7.13 & 3.41 & 0.48 & 7.80 & 5.12 & 0.66 \\
1.27 & 0.68 & 6.54 & 3.52 & 0.54 & 8.67 & 5.57 & 0.64 \\
1.81 & 0.21 & 6.49 & 3.60 & 0.55 & 9.63 & 5.17 & 0.54 \\
1.81 & 0.43 & 6.03 & 3.02 & 0.50 & 8.25 & 4.77 & 0.58 \\
\hline
\end{tabular}

level of significance are highlighted in bold cases.

Fig. 16 and Table 3 show that both the Lognormal and the Inverse Gaussian distributions are very similar. Since the statistics calculated for the Inverse Gaussian distribution always present lower values, as well as higher probability levels (not shown here), it is the one that best fits the presently obtained experimental data for $v_{t}$.

Fig. 17 shows the output distributions of $l_{s} / D$ obtained from the model of Dukler and Hubbard (1975) compared with the present experimental data. The input data to the model are the pipe diameter, the flow conditions and the passage frequency of slugs (obtained directly from the experimental data).

Results for the mean, standard deviation and coefficient of variance of the experimental values of $l_{s} / D$ are shown in Table 4 , as compared with the values obtained from the D\&H model. In general, the predicted values of $l_{s} / D$ show a percentage error relative to the experiments of $18 \%$. The predicted values also overestimate $\sigma$ and $c_{v}$ by $47 \%$ and $27 \%$ respectively.

The bold numbers in Table 5 illustrate the distributions that best fit the experimental and predicted (D\&H model) histograms of $l_{s} / D$.

The measured values were best represented by Normal and Gamma distributions. For low mixture velocities, the Normal distribution seems to be the more appropriate, whereas for high mixture velocities the distributions follow a Gamma distribution behavior. Values predicted through the D\&H model follow a different behavior. In this case, and for most of the experimental conditions, the histograms are best represented by a Lognormal distribution. Concerning the high superficial velocities, distributions are best fitted by a Gamma distribution.

Predicted values of $\Delta P$ are shown in Fig. 18. Results for $d P / d x$ follow the same trend and for this reason are not shown here. Statistics for the fitted distributions are shown in Table 6. These results show that estimations of $d P$ and $d P / d x$ can be well represented through Lognormal or Inverse Gaussian distributions, much the same as the distributions of $v_{t}$ used for the inlet condition of the D\&H model (Fig. 16). As previously observed, no significant difference can be noted between the Lognormal and Inverse Gaussian behaviors.

\subsection{Sensitivity of the Dukler and Hubbard (1975) model to input data from} Ujang et al. (2006)

The experiments of Ujang et al. (2006) are used for a further and independent assessment of the sensitivity of the D\&H model to input data of $v_{t}$. The tests were carried out for air-water flow in a pipe with $37 \mathrm{~m}$ in length and internal diameter of $0.078 \mathrm{~m}$. Five different base flow conditions were studied, with superficial velocities ranging in the intervals $V_{L S}=0.22 \mathrm{~ms}^{-1}$ to $0.61 \mathrm{~ms}^{-1}$ and $V_{G S}=2.27 \mathrm{~ms}^{-1}$ to $2.55 \mathrm{~ms}^{-1}$. The experiments of Ujang et al. (2006) were particularly selected for they include data on both distributions of $v_{t}$ and $l_{s}$. The works of Van Hout et al. (2001) and Cook and Behnia (2000a) do not present data on the distribution of $v_{t}$.

The present comparison was made against the conditions defined in Fig. 19. The input frequency distributions were taken directly from the parameters of the log-normal distribution provided in the original work from data at positions $x=0$ and $30 \mathrm{~m}$.

The returned output values of $l_{s} / D, d P$ and $d P / d x$ are presented in Fig. 19, where the lines represent the distributions that were not rejected at the $5 \%$ level based on the Kolmogorov-Smirnov test.

Table 7 presents data for the mean, standard deviation and coefficient of variance from the log-normal experimental distribution in comparison to analogous values obtained from the predictions of the Dukler and Hubbard (1975) model. Typically, the calculated values of $l_{s} / D$ show a small percentage error relative to the experiments of the order of $1.5 \%$. The unit cell model also furnished a standard deviation of the predicted $l_{s} / D$ that was, on average, 2.3 times higher than the experimental results, while the coefficient of variance exceeded the experimental values by the same amount.

Regarding the mean values of $l_{s} / D$, the unit cell model has provided better agreement with the experimental data of Ujang et al. (2006) than with the present experimental results. On the other hand, the dataset of Ujang et al. (2006) resulted in much larger values of the predicted standard deviation and coefficients of variance (see Table 4).

The statistics for the Kolmogorov-Smirnov test of the fitted distributions of predicted values of $l_{s} / D, d P$ and $d P / d x$ are shown in Table 8. The predicted lengths of liquid slugs follow a Log-normal distribution, but a Gamma distribution has also shown good agreement for two out of three experimental conditions. Distributions of $d P$ and $d P / d x$ are also satisfactorily represented by either Log-normal or Inverse-Gaussian distributions. Fig. 19 shows that only small differences can be noted between Log-normal and Inverse-Gaussian distributions. The behavior of the Gamma distributions is observed in Fig. 19b, c, d, f, g.

\subsection{Sensitivity of the Dukler and Hubbard (1975) model to the input coefficient of variance $c_{v}$}

The behavior of both $c_{v}\left(\Delta P / l_{u}\right)$ and $c_{v}\left(l_{s} / D\right)$ (as predicted through the D\&H model) for a varying $c_{v}\left(\nu_{t}\right)$ is shown in Fig. 20. Results show that both parameters vary linearly with $c_{v}\left(v_{t}\right)$. However, changes in

Table 5

Kolmogorov-Smirnov test statistics of the fitted experimental distributions of $l_{s} / D$ in comparison to results obtained through the D\&H model.

\begin{tabular}{|c|c|c|c|c|c|c|c|c|c|}
\hline \multicolumn{2}{|l|}{ Exp. Cond. } & \multicolumn{4}{|c|}{ K-S test, fit of exp. $l_{S} / D$} & \multicolumn{4}{|c|}{ K-S test, fit of D\&H model } \\
\hline$V_{L S}\left(\mathrm{~ms}^{-1}\right)$ & $V_{G S}\left(\mathrm{~ms}^{-1}\right)$ & Log-normal & Normal & Gamma & Inverse Gaussian & Log-normal & Normal & Gamma & Inverse Gaussian \\
\hline 0.72 & 0.27 & 0.19 & 0.14 & 0.18 & 0.22 & 0.07 & 0.14 & 0.07 & 0.07 \\
\hline 0.72 & 0.50 & 0.17 & 0.09 & 0.13 & 0.24 & 0.06 & 0.16 & 0.10 & 0.06 \\
\hline 0.72 & 0.78 & 0.17 & 0.09 & 0.15 & 0.20 & 0.07 & 0.11 & 0.06 & 0.09 \\
\hline 1.27 & 0.22 & 0.08 & 0.09 & 0.05 & 0.09 & 0.07 & 0.10 & 0.06 & 0.08 \\
\hline 1.27 & 0.42 & 0.08 & 0.07 & 0.08 & 0.10 & 0.07 & 0.11 & 0.06 & 0.08 \\
\hline 1.27 & 0.68 & 0.07 & 0.10 & 0.04 & 0.10 & 0.03 & 0.11 & 0.05 & 0.03 \\
\hline 1.81 & 0.21 & 0.08 & 0.08 & 0.05 & 0.11 & 0.11 & 0.06 & 0.08 & 0.13 \\
\hline 1.81 & 0.43 & 0.08 & 0.08 & 0.06 & 0.10 & 0.04 & 0.09 & 0.03 & 0.05 \\
\hline
\end{tabular}



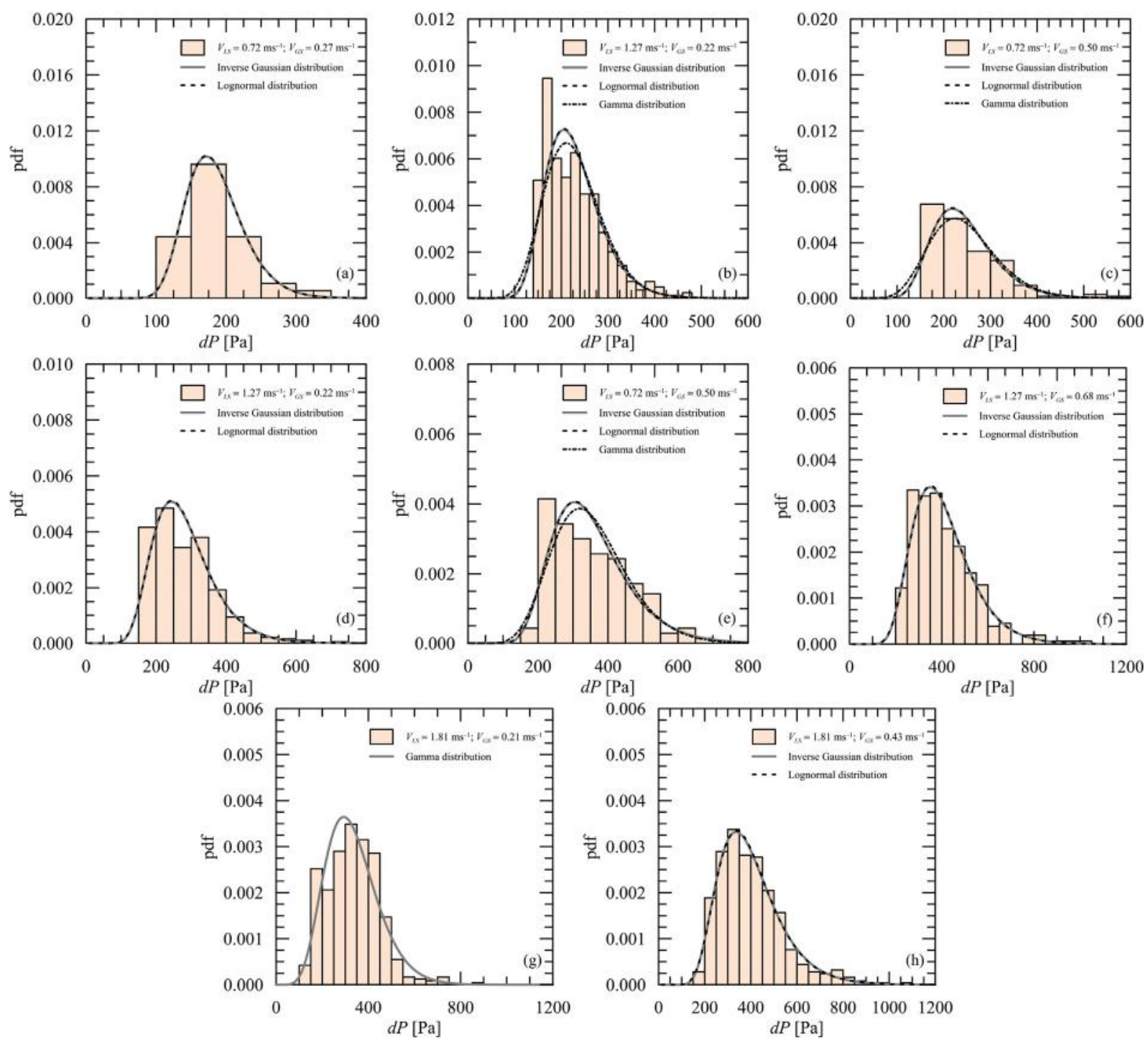

Fig. 18. Distributions of $d P$ (model of $\mathrm{D} \& \mathrm{H})$ at $x=9 \mathrm{~m}$ from the inlet position (present experiments).

Table 6

Kolmogorov-Smirnov test statistic of the fitted distributions to the pressure change and pressure gradient estimated from the D\&H model.

\begin{tabular}{|c|c|c|c|c|c|c|c|c|c|}
\hline \multicolumn{2}{|l|}{ Exp. Cond. } & \multicolumn{4}{|c|}{$\mathrm{K}-\mathrm{S}$ test, $d P$ from $\mathrm{D} \& \mathrm{H}$} & \multicolumn{4}{|c|}{ K-S test, $d P / d x$ from $D \& H$} \\
\hline$V_{L S}\left(\mathrm{~ms}^{-1}\right)$ & $V_{G S}\left(\mathrm{~ms}^{-1}\right)$ & Log-normal & Normal & Gamma & Inverse Gaussian & Log-normal & Normal & Gamma & Inverse Gaussian \\
\hline 0.72 & 0.27 & 0.10 & 0.14 & 0.11 & 0.10 & 0.08 & 0.15 & 0.10 & 0.08 \\
\hline 0.72 & 0.50 & 0.12 & 0.15 & 0.12 & 0.12 & 0.08 & 0.11 & 0.08 & 0.08 \\
\hline 0.72 & 0.78 & 0.06 & 0.10 & 0.07 & 0.06 & 0.11 & 0.14 & 0.11 & 0.11 \\
\hline 1.27 & 0.22 & 0.07 & 0.10 & 0.08 & 0.08 & 0.11 & 0.16 & 0.11 & 0.11 \\
\hline 1.27 & 0.42 & 0.06 & 0.12 & 0.09 & 0.06 & 0.10 & 0.14 & 0.11 & 0.10 \\
\hline 1.27 & 0.68 & 0.05 & 0.10 & 0.06 & 0.05 & 0.06 & 0.09 & 0.06 & 0.06 \\
\hline 1.81 & 0.21 & 0.07 & 0.07 & 0.05 & 0.08 & 0.17 & 0.22 & 0.18 & 0.17 \\
\hline 1.81 & 0.43 & 0.02 & 0.08 & 0.04 & 0.03 & 0.07 & 0.10 & 0.07 & 0.07 \\
\hline
\end{tabular}

The distributions that were not rejected at the $5 \%$ level of significance are highlighted in bold cases.

$c_{v}\left(\nu_{t}\right)$ are specially relevant to predictions of $l_{s} / D$. A coefficient of variation of 0.6 for $\nu_{t}$ corresponds to a $c_{v}$ of 0.11 for $\Delta P / l_{u}$. On the other hand, for the same $c_{v}\left(\nu_{t}\right)(=0.6)$, a value of 1.2 is found for $c_{v}\left(l_{s} / D\right)$. Thus, relatively large variations on the prediction of $v_{t}$ have a small effect on the determination of $\Delta P / l_{u}$ but a large effect on $l_{s} / D$.

The present experiments and the data of Ujang et al. (2006) tend to corroborate this finding (Fig. 21). Despite the large scatter in the data, the coefficient of variance of $l_{s} / D$ is consistently higher than the coefficient of variance of $\Delta P / l_{u}$ for a given $v_{t}$. According to the experiments, a $c_{v}\left(v_{t}\right)=0.6$ corresponds to $c_{v}\left(\Delta P / l_{u}\right)=0.64$ (present) and 0.25 (Ujang et al., 2006) and $c_{v}\left(l_{s} / D\right)=0.77$ (present) and 1.0 (Ujang et al., 2006).

\section{Slug flow predictions with evolution models}

The equation of Cook and Behnia (2000a) is investigated in this 

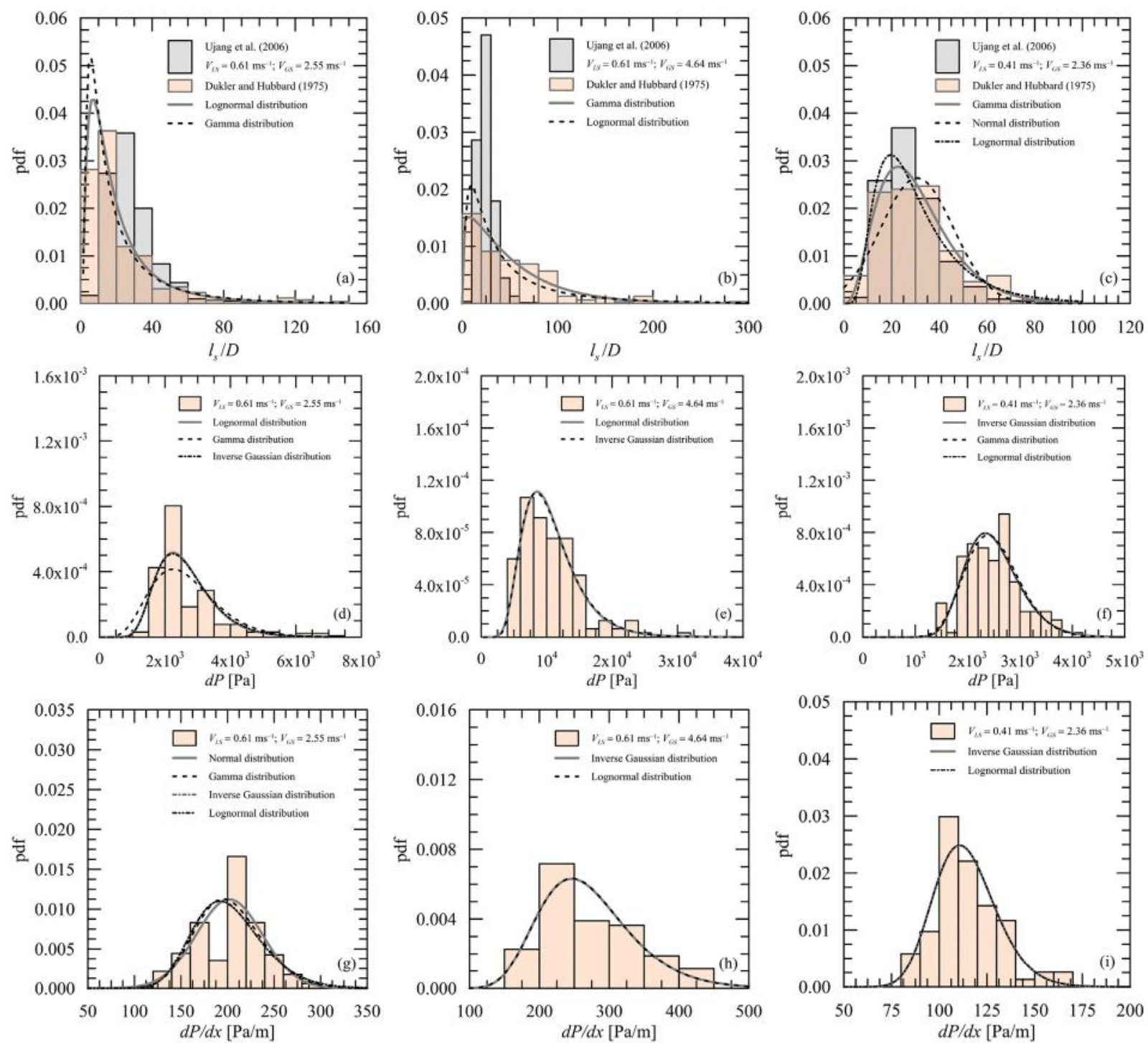

Fig. 19. Histograms (D\&H model) obtained through input Log-normal distributions of Ujang et al. (2006): $l_{s} / D, d P$ e $d P / d x$ at $x=30 \mathrm{~m}$. Lines denote the fitted distributions to the predicted data.

Table 7

Comparison between the experimental data of Ujang et al. (2006) for the mean, standard deviation and coefficient of variance of the slug length data $\left(l_{s} / D\right)$ and the corresponding predicted values by the $\mathrm{D} \& \mathrm{H}$ model.

\begin{tabular}{llllllll}
\hline Exp. Cond. & \multicolumn{3}{c}{$\left(l_{s} / D\right)_{\exp }$} & \multicolumn{5}{c}{$\left(l_{s} / D\right)_{D \& H}$} \\
\hline$V_{L S}\left(\mathrm{~ms}^{-1}\right)$ & $V_{G S}\left(\mathrm{~ms}^{-1}\right)$ & $\mu_{L N_{\exp }}$ & $\sigma_{L N_{\exp }}$ & $c_{\text {vexp }}$ & $\mu_{L N_{D H}}$ & $\sigma_{L N_{D H}}$ & $c_{v_{D H}}$ \\
\hline 0.61 & 2.55 & 3.22 & 0.44 & 0.14 & 2.73 & 0.91 & 0.33 \\
0.61 & 4.64 & 3.17 & 0.33 & 0.10 & 3.47 & 1.12 & 0.32 \\
0.41 & 2.36 & 3.24 & 0.41 & 0.13 & 3.29 & 0.56 & 0.17 \\
\hline
\end{tabular}

section. The analysis considers the evolution of mean values and standard deviation of liquid slug lengths $\left(l_{s} / D\right)$ and time intervals between subsequent slugs (T). The data of Ujang et al. (2006) and the present data are used for model validation.

About 10,000 unit cells are considered in the simulations. The time step is $0.005 \mathrm{~s}$. A convergence test showed that an increase in the number of cells or a decrease in the time step resulted in a variation of less than $1 \%$ in the mean length of the liquid slugs.

The model of Cook and Behnia (2000a) is observed to be extremely sensitive to the choice of the bubble propagation velocity relation. In
Table 8

Kolmogorov-Smirnov test statistics for $l_{s} / D, d P$ and $d P / d x$ distributions obtained by the D\&H model with input $v_{t}$ distributions based on the data of Ujang et al. (2006).

\begin{tabular}{llllll}
\hline Exp. Cond. & \multicolumn{5}{l}{ K-S test, $l_{s} / D$ from D\&H } \\
\hline$V_{L S}\left(\mathrm{~ms}^{-1}\right)$ & $V_{G S}\left(\mathrm{~ms}^{-1}\right)$ & Log-normal & Normal & Gamma & Inverse Gaussian \\
\hline 0.61 & 2.55 & $\mathbf{0 . 1 0}$ & 0.24 & 0.16 & $\mathbf{0 . 1 1}$ \\
0.61 & 4.64 & $\mathbf{0 . 1 0}$ & 0.15 & $\mathbf{0 . 0 6}$ & 0.17 \\
0.41 & 2.36 & $\mathbf{0 . 1 0}$ & $\mathbf{0 . 0 8}$ & $\mathbf{0 . 0 7}$ & 0.13 \\
\hline Exp. Cond. & \multicolumn{5}{l}{} \\
\hline 0.61 & 2.55 & $\mathbf{0 . 1 7}$ & 0.22 & $\mathbf{0 . 1 7}$ & 0.18 \\
0.61 & 4.64 & $\mathbf{0 . 0 7}$ & 0.12 & 0.09 & $\mathbf{0 . 0 7}$ \\
0.41 & 2.36 & $\mathbf{0 . 0 6}$ & $\mathbf{0 . 0 7}$ & $\mathbf{0 . 0 6}$ & $\mathbf{0 . 0 6}$ \\
\hline Exp. Cond. & & K-S test, $d P / d x$ from D\&H & \\
\cline { 4 - 5 } 0.61 & 2.55 & 0.15 & $\mathbf{0 . 1 2}$ & $\mathbf{0 . 1 3}$ & 0.15 \\
0.61 & 4.64 & $\mathbf{0 . 0 9}$ & 0.12 & 0.10 & $\mathbf{0 . 0 9}$ \\
0.41 & 2.36 & $\mathbf{0 . 0 8}$ & 0.11 & 0.09 & $\mathbf{0 . 0 8}$ \\
\hline
\end{tabular}

The distributions that were not rejected at the $5 \%$ level of significance are highlighted in bold cases. 

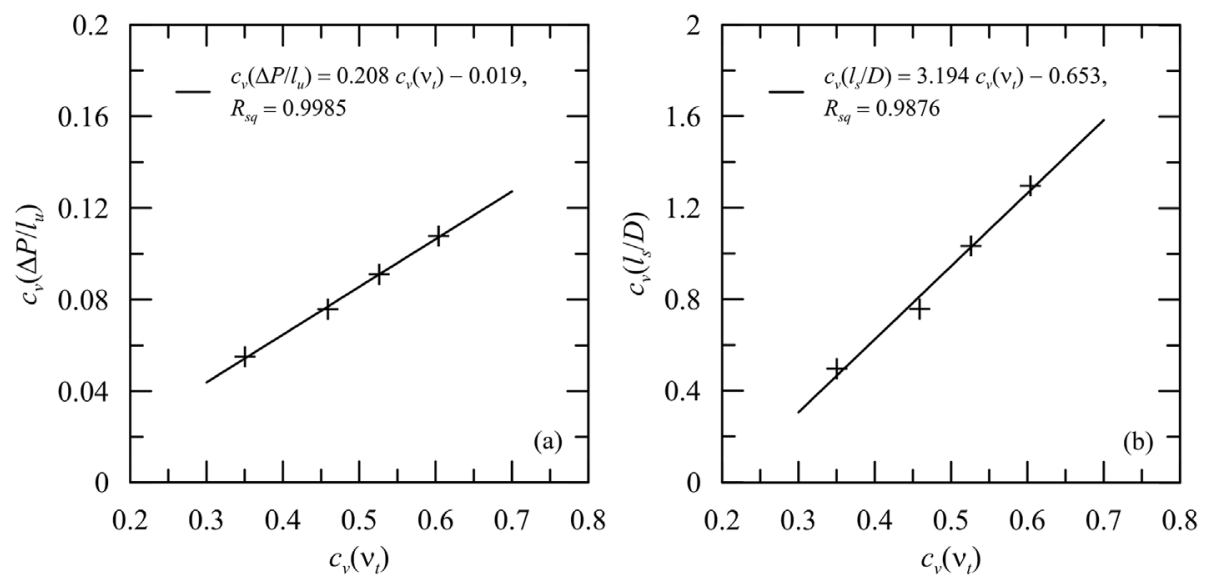

Fig. 20. Behavior of the coefficients of variance of $\Delta P / l_{u}$ and $l_{s} / D$ as a function of the coefficient of variance of $v_{t}$.
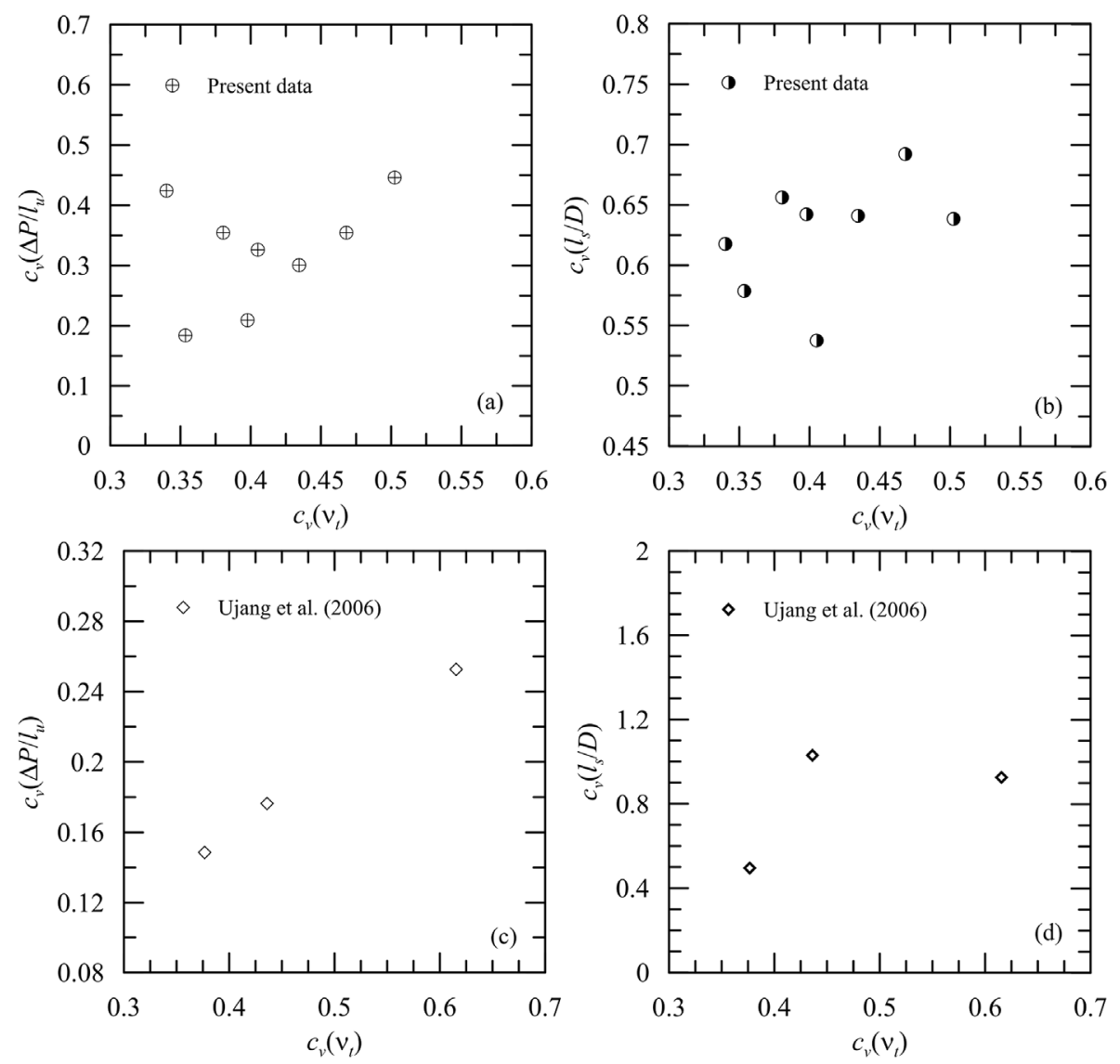

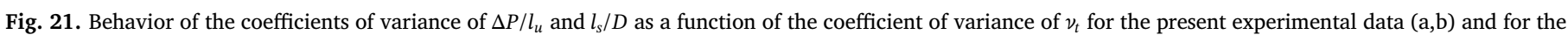
data of Ujang et al. (2006) (c,d).

fact, the sensitivity of this important closure parameter is known to be deficiently discussed in literature.

Fig. 22 shows a comparison between the mean and the root-mean square values of the liquid slug lengths and time interval between slugs obtained from the Cook and Behnia (2000a) model and the data of Ujang et al. (2006).

One clear tendency of the computations is to smoothly increase the slug lengths along the distance from the inlet. For the flow conditions of $V_{L S}=0.61 \mathrm{~ms}^{-1}$ and $V_{G S}=2.55 \mathrm{~ms}^{-1}$ and $4.64 \mathrm{~ms}^{-1}$, the mean relative error between predictions and experimental results was $23 \%$ and $18 \%$, respectively. The lower liquid velocity condition provided a mean relative error of $35.5 \%$. Fig. 22a shows that up to $10 \mathrm{~m}$, the experimental and the numerical data are relatively close. However, at the last position $(x=30 \mathrm{~m})$ the difference between experiments and numerical prediction were as high as $46 \%$.

The standard deviation of predicted values of $l_{s} / D$ are shown in Fig. 22b. The experimental data present a nearly linear growth along the pipe length. This tendency is not predicted by the Cook and Behnia 

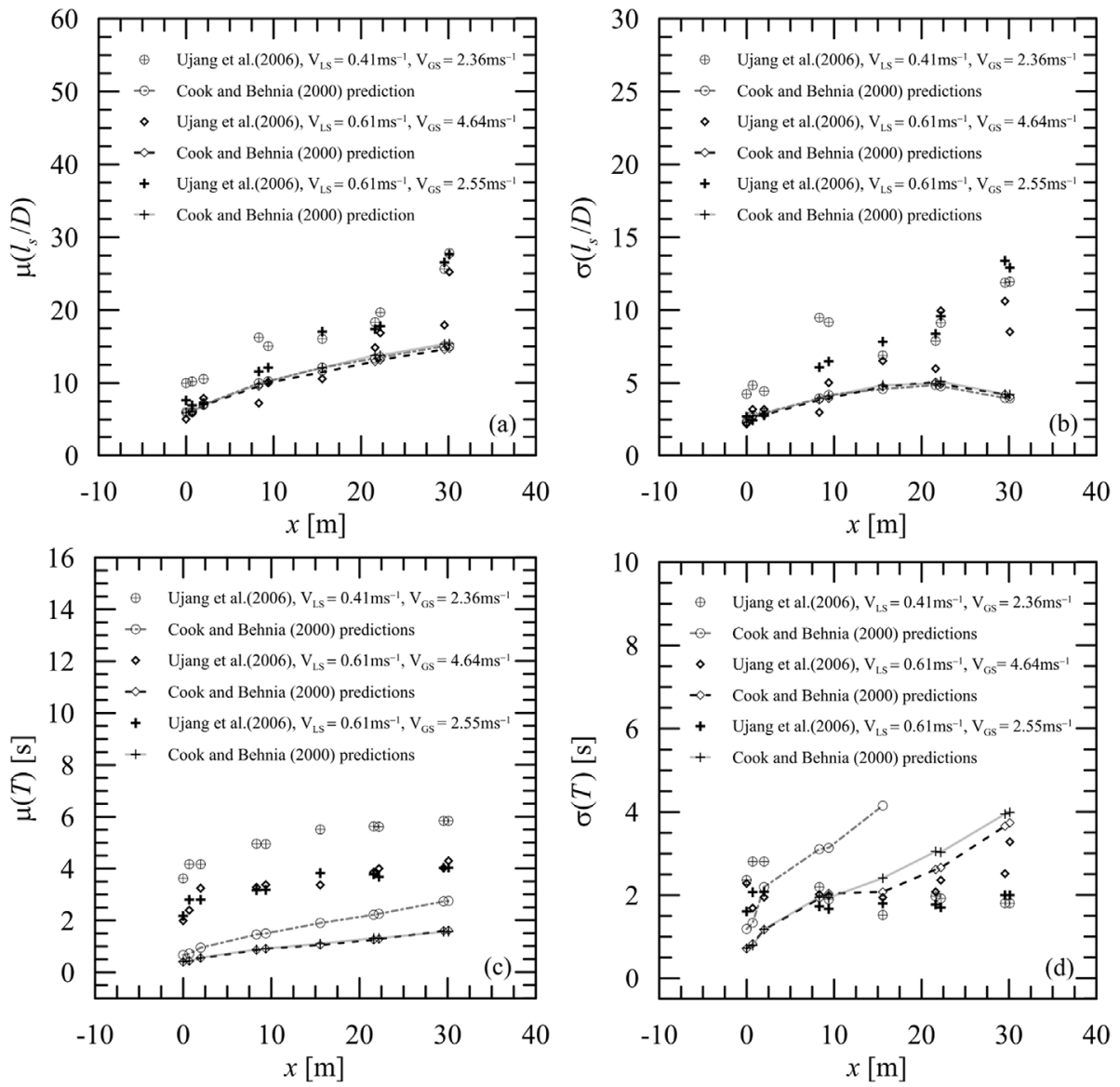

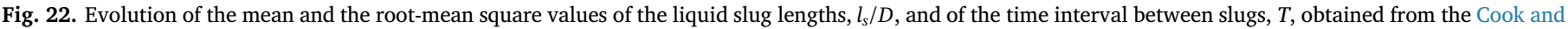
Behnia (2000a) model in comparison to the data of Ujang et al. (2006).

(2000a) model, which shows a smooth growth of $\sigma\left(l_{s} / D\right)$ up to $23 \mathrm{~m}$, followed by a slight decrease at $x=30 \mathrm{~m}$, where relative errors are as high as $67 \%$.

Due to the merging process, and as expected, the experiments show that the time interval between slugs $(T)$ increases with the downstream distance and eventually reaches a stable state (Fig. 22c). The predicted behavior follows the same trend, but relative errors are of the order of $70 \%$. The standard deviation of $T$ shows an almost constant value along the pipe length (Fig. 22d). Theoretical predictions tend to increase linearly with distance from the pipe inlet.

The above discussion is further illustrated with an analysis of the experimental distributions of $v_{t}$ obtained in the present work (Fig. 23). The overall agreement between the theoretical predictions and the experimental data is reasonable, as shown also in Table 9. Agreement of theory with the experimental data is poor for the low liquid velocity conditions. For the other experimental conditions, predicted mean values of $v_{t}$ lie within $15 \%$ of the experimental data.

Fig. 23 also shows the suggested distributions to the values of $v_{t}$ obtained through the model of C\&B. The statistics for the distributions that were not rejected at the $5 \%$ level based on the KolmogorovSmirnov test are highlighted in bold cases in Table 10. Note that the C\& B model correctly predicts the behavior of $v_{t}$ according to Log-normal or Inverse Gaussian distributions.

Fig. 24 compares distributions of $l_{s} / D$ obtained from the model of $\mathrm{C}$ $\& \mathrm{~B}$ with the present experimental data. Despite the clear changes in $l_{s} / D$ with the experimental conditions, the predictions showed insensitivity with changes in the input gas and liquid superficial velocities. The model of Cook and Behnia (2000a) tends to furnish $l_{s} / D=13$ for all present experimental conditions, as illustrated in Table 11. The predicted coefficient of variance for $l_{s} / D$ is approximately $22 \%$ smaller that the experimental value.

The statistics for the distributions of $l_{s} / D$ that were not rejected at the $5 \%$ level based on the Kolmogorov-Smirnov test are highlighted in bold cases in Table 12. Differently from the Dukler and Hubbard model, the equation of Cook and Behnia (2000a) provided estimates for the slug length distributions that followed either a Normal or Gamma distributions. The results are in good agreement with experimental results, as shown in Table 5. In summary, although the equation of Cook and Behnia does not correctly predicts the mean value of slug lengths, it does reflect the correct behavior of $l_{s} / D$ distribution.

The above results suggest that the C\&B model may be used to generate input distributions of $T\left(=v_{t}^{-1}\right)$ to be used in unit-cell models. On the other hand, the percentage error of $l_{s} / D$ found with a directly application of the model of Cook and Behnia (2000a) is 77\%.

Results on predictions of the mean values of $v_{t}, l_{s} / D$ and $d P / d x$ are consolidated in Fig. 25. The general agreement with the data for the predictions of $v_{t}$ (through C\&B model) is very good (Fig. 25a). The increase in $V_{G S}$ for a fixed $V_{L S}$ always results in a decrease of $l_{S} / D$. However, the model of C\&B is not capable of capturing this change. The model tends to furnish the same value of $l_{s} / D$ for the several experimental conditions. Computations based on the D\&H model, however, 

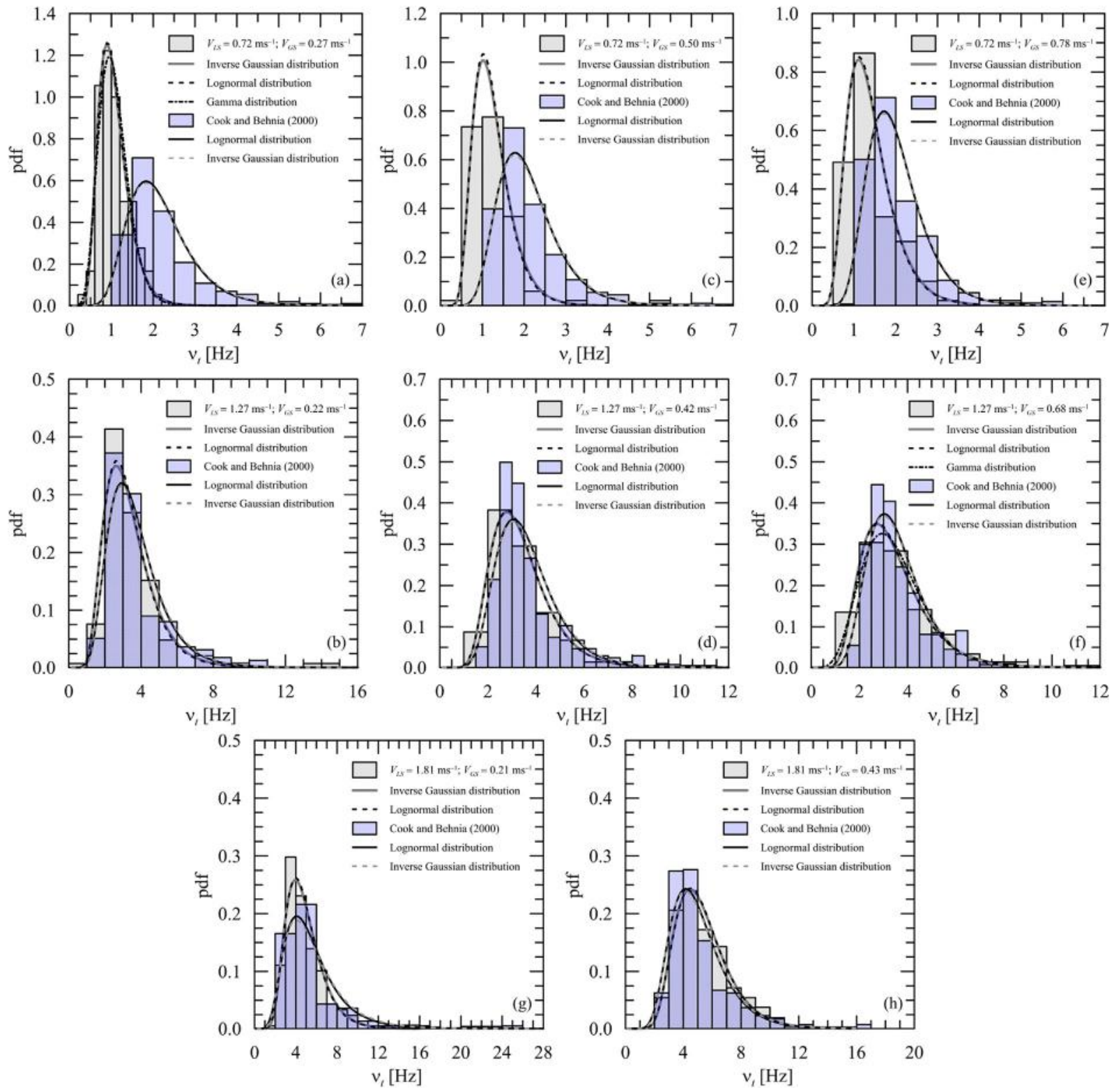

Fig. 23. Distributions of $v_{t}$ according to the model of Cook and Behnia (2000a) against the present experimental data.

Table 9

Comparison between the experimental values of the mean, standard deviation and coefficient of variance of $\nu_{t}$ and the corresponding predicted values by the model of Cook and Behnia (2000a).

\begin{tabular}{llllllll}
\hline Exp. Cond & & \multicolumn{2}{c}{$\nu_{t}$ (present data) } & \multicolumn{2}{c}{$v_{t}$ from Cook and Behnia (2000a) } \\
\hline$V_{L S}\left(\mathrm{~ms}^{-1}\right)$ & $V_{G S}\left(\mathrm{~ms}^{-1}\right)$ & $\mu_{\exp }$ & $\sigma_{\exp }$ & $c_{\text {vexp }}$ & $\mu_{C B}$ & $\sigma_{C B}$ & $c_{v_{C B}}$ \\
\hline 0.72 & 0.27 & 1.06 & 0.36 & 0.34 & 2.20 & 0.89 & 0.41 \\
0.72 & 0.50 & 1.25 & 0.58 & 0.47 & 2.12 & 0.84 & 0.40 \\
0.72 & 0.78 & 1.41 & 0.61 & 0.43 & 2.03 & 0.78 & 0.38 \\
1.27 & 0.22 & 3.36 & 1.69 & 0.50 & 3.73 & 1.71 & 0.46 \\
1.27 & 0.42 & 3.38 & 1.29 & 0.38 & 3.67 & 1.48 & 0.40 \\
1.27 & 0.68 & 3.44 & 1.37 & 0.40 & 3.60 & 1.39 & 0.39 \\
1.81 & 0.21 & 4.86 & 1.97 & 0.41 & 5.67 & 3.65 & 0.64 \\
1.81 & 0.43 & 5.37 & 1.90 & 0.35 & 5.14 & 2.27 & 0.44 \\
\end{tabular}

tend to follow the changes (Fig. 25b). Results on pressure change are shown in Fig. 25c. The model of C\&B excessively over predicts the experimental data, for all gas flow rates. The model of D\&H performs well.
Table 10

Kolmogorov-Smirnov test statistics for the fitted distributions to $v_{t}$ estimated from the C\&B model.

\begin{tabular}{llllll}
\hline Exp. Cond. & \multicolumn{5}{c}{$v_{t}$ from C\&B } \\
\hline$V_{L S}\left(\mathrm{~ms}^{-1}\right)$ & $V_{G S}\left(\mathrm{~ms}^{-1}\right)$ & Log-normal & Normal & Gamma & Inverse Gaussian \\
\hline 0.72 & 0.27 & $\mathbf{0 . 0 9}$ & 0.15 & 0.11 & $\mathbf{0 . 0 9}$ \\
0.72 & 0.50 & $\mathbf{0 . 0 7}$ & 0.15 & 0.10 & $\mathbf{0 . 0 8}$ \\
0.72 & 0.78 & $\mathbf{0 . 0 9}$ & 0.14 & 0.11 & $\mathbf{0 . 0 9}$ \\
1.27 & 0.22 & $\mathbf{0 . 1 0}$ & 0.18 & 0.12 & $\mathbf{0 . 1 0}$ \\
1.27 & 0.42 & $\mathbf{0 . 1 2}$ & 0.19 & 0.14 & $\mathbf{0 . 1 2}$ \\
1.27 & 0.68 & $\mathbf{0 . 0 9}$ & 0.16 & 0.12 & $\mathbf{0 . 1 0}$ \\
1.81 & 0.21 & $\mathbf{0 . 1 5}$ & 0.25 & 0.19 & $\mathbf{0 . 1 7}$ \\
1.81 & 0.43 & $\mathbf{0 . 0 9}$ & 0.18 & 0.12 & $\mathbf{0 . 1 0}$ \\
\hline
\end{tabular}

\section{Final remarks}

The present work shows how a distinction between the physical and feasible domains is important in the numerical implementation of models. Models that are expected to be applicable to certain conditions, 

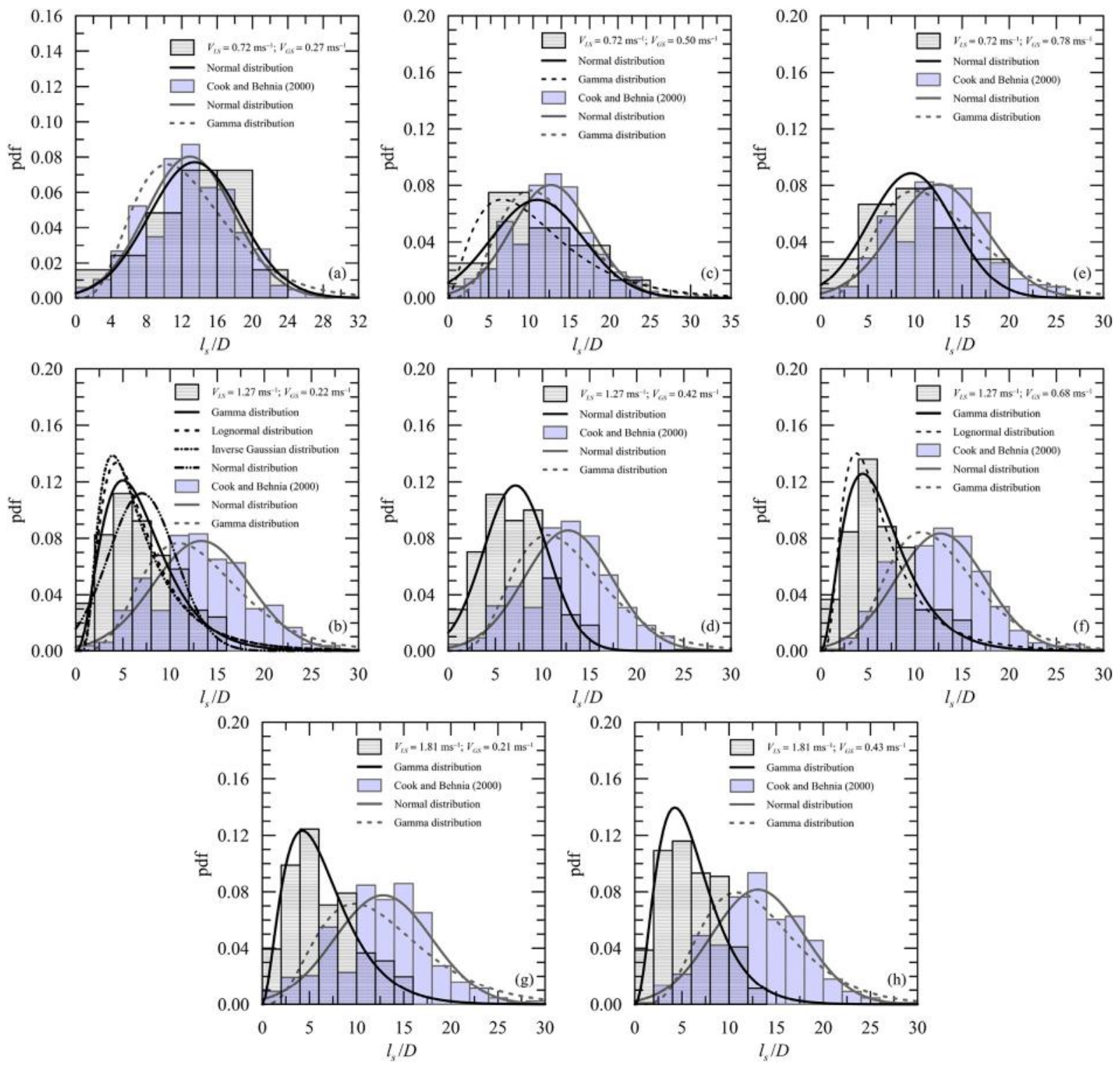

Fig. 24. Distributions of $l_{s} / D$ according to the model of C\&B against the present experimental data.

Table 11

Comparison between the experimental values of the mean, standard deviation and coefficient of variance of the slug length data $\left(l_{s} / D\right)$ and the corresponding predicted values by the C\&B model.

\begin{tabular}{llllllll}
\hline Exp. Cond & & \multicolumn{3}{c}{$l_{S} / D$ (present data) } & \multicolumn{5}{c}{$l_{S} / D$ from C\&B } \\
\hline$V_{L S}\left(\mathrm{~ms}^{-1}\right)$ & $V_{G S}\left(\mathrm{~ms}^{-1}\right)$ & $\mu_{\exp }$ & $\sigma_{\exp }$ & $c_{v_{\exp }}$ & $\mu_{C B}$ & $\sigma_{C B}$ & $c_{v_{C B}}$ \\
\hline 0.72 & 0.27 & 13.45 & 5.26 & 0.39 & 12.92 & 4.97 & 0.38 \\
0.72 & 0.50 & 11.02 & 5.81 & 0.53 & 12.72 & 4.97 & 0.39 \\
0.72 & 0.78 & 9.60 & 4.55 & 0.47 & 12.61 & 4.94 & 0.39 \\
1.27 & 0.22 & 7.02 & 3.58 & 0.51 & 13.32 & 5.11 & 0.38 \\
1.27 & 0.42 & 7.13 & 3.41 & 0.48 & 12.72 & 4.67 & 0.37 \\
1.27 & 0.68 & 6.54 & 3.52 & 0.54 & 12.72 & 4.78 & 0.38 \\
1.81 & 0.21 & 6.49 & 3.60 & 0.55 & 12.82 & 5.15 & 0.40 \\
1.81 & 0.43 & 6.03 & 3.02 & 0.50 & 13.06 & 4.90 & 0.38 \\
\hline
\end{tabular}

may not be so due to some unjustifiable mathematical operation. The model of Dukler and Hubbard (1975) was shown not to furnish mathematical solutions for flows with $V_{G S}>6 \mathrm{~ms}^{-1}$.

The slug flow models of Cook and Behnia (2000a) and Dukler and Hubbard (1975) can be combined to provide predictions on slug length
Table 12

Kolmogorov-Smirnov test statistics for the fitted distributions of the slug length $\left(l_{s} / D\right)$ as estimated from the C\&B model.

\begin{tabular}{llllll}
\hline Exp. Cond. & \multicolumn{5}{c}{$l_{S} / D$ from C\&B. } \\
\hline$V_{L S}\left(\mathrm{~ms}^{-1}\right)$ & $V_{G S}\left(\mathrm{~ms}^{-1}\right)$ & Log-normal & Normal & Gamma & Inverse Gaussian \\
\hline 0.72 & 0.27 & 0.13 & $\mathbf{0 . 0 5}$ & $\mathbf{0 . 0 9}$ & 0.20 \\
0.72 & 0.50 & 0.14 & $\mathbf{0 . 0 4}$ & $\mathbf{0 . 1 0}$ & 0.18 \\
0.72 & 0.78 & 0.13 & $\mathbf{0 . 0 5}$ & $\mathbf{0 . 0 9}$ & 0.21 \\
1.27 & 0.22 & 0.11 & $\mathbf{0 . 0 4}$ & $\mathbf{0 . 0 7}$ & 0.14 \\
1.27 & 0.42 & 0.13 & $\mathbf{0 . 0 4}$ & $\mathbf{0 . 0 9}$ & 0.16 \\
1.27 & 0.68 & 0.10 & $\mathbf{0 . 0 5}$ & $\mathbf{0 . 0 8}$ & 0.11 \\
1.81 & 0.21 & 0.17 & $\mathbf{0 . 0 5}$ & $\mathbf{0 . 1 3}$ & 0.24 \\
1.81 & 0.43 & 0.11 & $\mathbf{0 . 0 3}$ & $\mathbf{0 . 0 8}$ & 0.14 \\
\end{tabular}

distributions. The combination of these approaches avoids the need to resort to an empirical expression for $\nu_{t}$. The idea is to use the evolution model of Cook and Behnia (2000a) to generate a frequency distribution to be used as an input parameter in the mechanistic model of Dukler and Hubbard (1975). The predicted distributions of pressure drop and slug length are shown to be a good match to experimental data. 


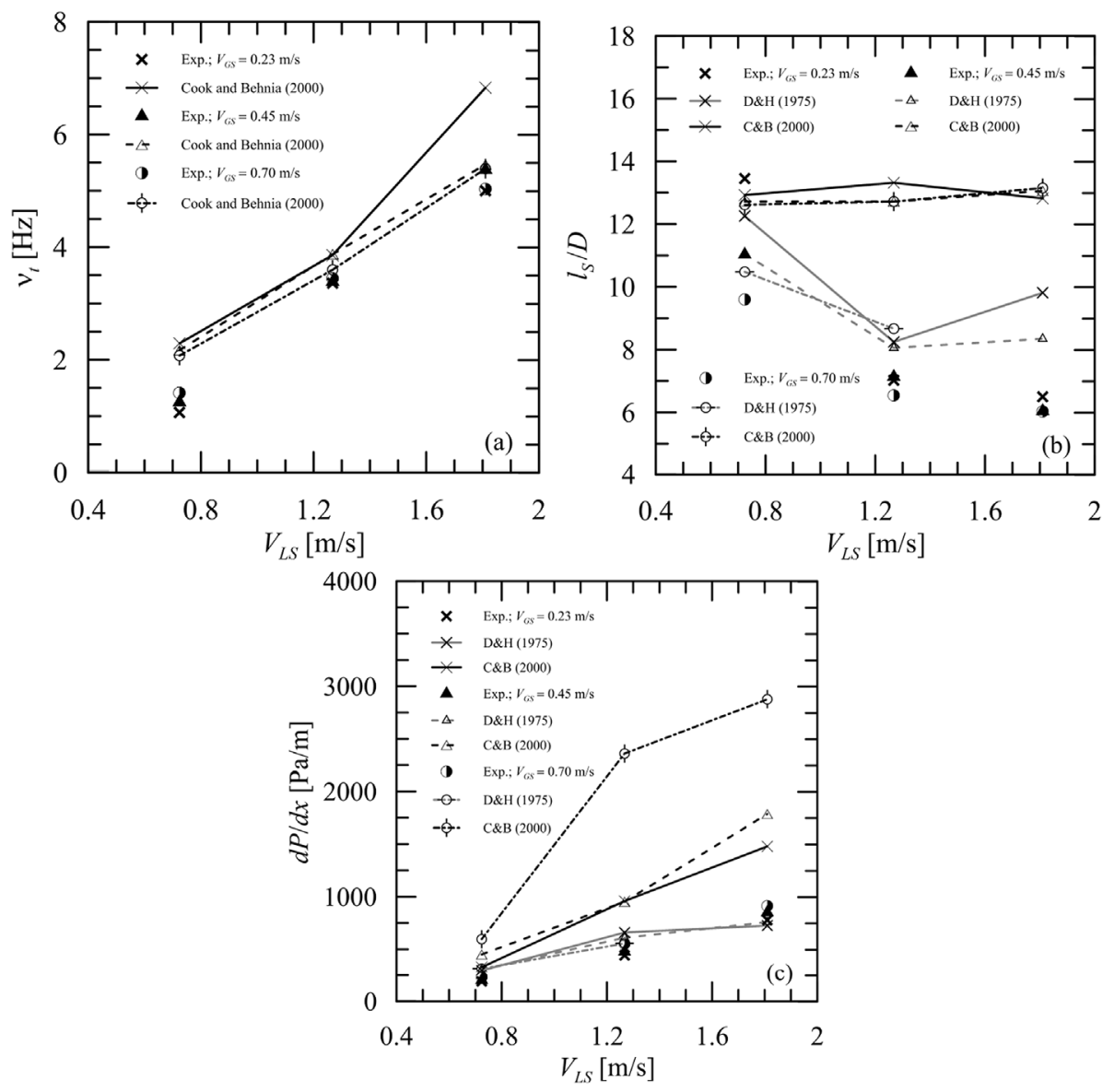

Fig. 25. Comparison with the present experimental data of predictions of $v_{t}, l_{s} / D$ and $d P / d x$.

\section{Acknowledgements}

GFNG is thankful to CAPES for his research scholarship. APSF is grateful to the Brazilian National Research Council (CNPq) for the award of a Research Fellowship (No 305338/2014-5). JBRL benefited from a CNPq Research Fellowship (Grant No 309455/2016-2) and from further financial support through Grants CNPq 458249/2014-9 and FAPERJ E-26/203.257/2016.

\section{References}

Al-Safran, E.M., 2009. Investigation and prediction of slug frequency in gas/liquid horizontal pipe flow. J. Petroleum Sci. Eng. 69, 143-155.

Al-Safran, E.M., 2016. Probabilistic modeling of slug frequency in gas/liquid pipe flow using the Poisson probability theory. J. Petroleum Sci. Eng. 138, 88-96.

Al-Safran, E.M., Taitel, Y., Brill, J.P., 2004. Prediction of slug length distribution along a hilly terrain pipeline using slug tracking Model. J. Energy Resour. Technol. 126, 54-62.

Al-Safran, E.M., Sarica, C., Zhang, H.Q., Brill, J.P., 2005. Probabilistic/mechanistic modeling of slug length distribution in a horizontal pipeline. SPE Prod. Facil. (May) 5-8.

Al-Safran, E.M., Kouba, G., Brill, J.P., 2013. Statistical analysis and hydrodynamic modeling of unsteady gas-liquid slug velocity behavior. J. Petroleum Sci. Eng. 109, $155-163$.

Bandeira, F.J.S., Goncalves, G.F.N., Loureiro, J.B.R., Silva Freire, A.P., 2017. Turbulence and bubble break up in slug flow with wall injection. Flow, Turbul. Combust. 98, 923-945.

Barnea, D., Taitel, Y., 1993. A model for slug length distribution in gas-liquid slug flow. Int. J. Multiph. Flow 19 (5), 829-838.

Brill, J.P., Schmidt, Z., Coberly, W.A., Herring, J.D., Moore, D.W., 1981. Analysis of twophase tests in large diameter flow lines in Prudhoe Bay Field. SPE J. 8305, 363-377.

Cook, M., Behnia, M., 2000a. Slug length prediction in near horizontal gas-liquid intermittent flow. Chem. Eng. Sci. 55, 2009-2018.

Cook, M., Behnia, M., 2000b. Pressure drop calculation and modelling of inclined intermittent gas and liquid flow. Chem. Eng. Sci. 55, 4699-4708.

Davies, R.M., Taylor, G.I., 1950. The mechanics of large bubbles rising through extended liquids and through liquids in tubes. Proc. Roy. Soc. 200 A, 375-390.
Dhulesia, H., Bernicot, M., Deheuvels, P., 1991. Statistical analysis and modelling of slug lengths. In: $5^{\text {th }}$ International Conference on Multiphase Production, pp. 80-112.

Dukler, A.E., Hubbard, M.G., 1975. A model for gas-liquid slug flow in horizontal and near horizontal tubes. Ind. Eng. Chem. Fund. 14 (4), 337-347.

Dumitrescu, D.T., 1943. Stromung an einer luftblase im senkrechten Rohr. Z. Angew. Math. Mech. 23, 139-149.

Fabre, J., Liné, A., 1992. Modeling of two-phase slug flow. Ann. R. Fluid Mech. 24 (1), $21-46$.

Gregory, G.A., Scott, D.S., 1969. Correlation of liquid slug velocity and frequency in horizontal cocurrent gas-liquid slug flow. AIChE J. 15, 933-935.

Heywood, N.I., Richardson, J.F., 1979. Slug flow of air-water mixtures in a horizontal pipe: determination of liquid holdup by $\gamma$-ray absorption. Chem. Eng. Sci. 17-30.

JCGM, 2010. Evaluation of measurement data - Guide to the expression of uncertainty in measurement. Joint Committee for Guides in Metrology. JCGM 100, 2008 Corrected version 2010. 134 pp.

Magalhaes, G.R., Goncalves, G.F.N., Loureiro, J.B.R., Silva Freire, A.P., 2013. An experimental investigation of the effects of gas solubility on the properties of horizontal slug flow. Int. J. Multiph. Flow 50, 33-40.

Mandhane, J.M., Gregory, G.A., Aziz, K., 1974. A flow pattern map for gas-liquid flow in horizontal pipes. Int. J. Multiph. Flow. 1, 537-553.

Manolis, I.G., Mendes-Tatsis, M.A., Hewitt, G.F., 1995. The effect of pressure on slug frequency on two-phase horizontal flow. In: $2^{\text {th }}$ International Conference on Multiphase Flow.

Massey Jr., F.J., 1951. The Kolmogorov-Smirnov test for goodness of fit. J. Am. Stat. Assoc. 46 (253), 68-78.

Matamoros, L.M.C., Loureiro, J.B.R., Silva Freire, A.P., 2014. Length-area-volume of long bubbles in horizontal slug flow. Int. J. Multiph. Flow. 65, 24-30.

Moissis, R., Griffith, P., 1962. Entrance effects in a two phase slug flow. J. Heat. Transf. 84, 29-39.

Nicholson, M.K., Aziz, K., Gregory, G.A., 1978. Intermittent two phase flow in horizontal pipes: predictive models. Can. J. Chem. Eng. 56, 653-663.

Nicklin, D.J., Wilkes, J.O., Davidson, J.F., 1962. Two-phase flow in vertical tubes. Trans. Inst. Chem. Eng.

Nieckele, A.O., Carneiro, J.N.E., Chucuya, R.C., Azevedo, J.H.P., 2013. Initiation and statistical evolution of horizontal slug flow with a two-fluid model. J. Fluids Eng. 135 (12), 121302.

Nogueira, S., Sousa, R.G., Pinto, A.M.F.R., Riethmuller, M.L., Campos, J.B.L.M., 2003. Simultaneous PIV and pulsed shadow technique in slug flow: a solution for optical problems. Exp. Fluids 35, 598-609.

Nydal, O.J., Pintus, S., Andreussi, P., 1992. Statistical characterization of slug flow in 
horizontal pipes. Int. J. Multiph. Flow. 18 (3), 439-453.

Orell, A., 2005. Experimental validation of a simple model for gas-liquid slug flow in horizontal pipes. Chem. Eng. Sci. 60 (5), 1371-1381.

Taitel, Y., Barnea, D., 1990. A consistent approach for calculating pressure drop in inclined slug flow. Chem. Eng. Sci. 5, 1199-1206.

Taitel, Y., Dukler, A.E., 1976. A model for predicting flow regime transitions in horizontal and near horizontal gas-liquid flow. AIChE J. 22, 47-55.

Taitel, Y., Sarica, C., Brill, J.P., 2000. Slug flow modeling for downward inclined pipe flow: theoretical considerations. Int. J. Multiph. Flow. 26, 833-844.

Ujang, P.M., Lawrence, C.J., Hale, C.P., Hewitt, G.F., 2006. Slug initiation and evolution in two-phase horizontal flow. Int. J. Multiph. Flow. 32 (5), 527-552.

Van Hout, R., Barnea, D., Shemer, L., 2001. Evolution of statistical parameters of gasliquid slug flow along vertical pipes. Int. J. Multiph. Flow. 27.

Wallis, G.B., 1969. One Dimensional Two-phase Flow. McGraw-Hill, New York 409p. Zabaras, G.B., 2000. Prediction of slug frequency for gas/liquid flows. SPE J. 5 (3),

$$
\text { 252-258. }
$$

Zhang, H.Q., Al-Safran, E.M., Jayawardena, S.S., Redus, C.L., Sarica, C., Brill, J.P., 2003 Modeling of slug dissipation and generation in gas-liquid hilly-terrain pipe flow. J. Energy Resour. Technol. 125, 161-168. 